\title{
Dorsal Horn Gastrin-Releasing Peptide Expressing Neurons Transmit Spinal Itch But Not Pain Signals
}

\author{
Gioele W. Albisetti, ${ }^{1,2}$ Martina Pagani, ${ }^{1,2}$ Evgenia Platonova, ${ }^{3}$ Ladina Hösli, ${ }^{1,2}$ Helge C. Johannssen, ${ }^{1}$ \\ (D)Jean-Marc Fritschy, ${ }^{1,2}$ (D)Hendrik Wildner, ${ }^{1}$ and ${ }^{D}$ Hanns Ulrich Zeilhofer ${ }^{1,2,4,5}$ \\ ${ }^{1}$ Institute of Pharmacology and Toxicology, University of Zurich, CH-8057 Zurich, Switzerland, ${ }^{2}$ Neuroscience Center Zurich, CH-8057 Zurich, Switzerland, \\ ${ }^{3}$ Center for Microscopy and Image Analysis, University of Zurich, CH-8057 Zurich, Switzerland, ${ }^{4}$ Drug Discovery Network Zurich, CH-8057 Zurich, \\ Switzerland, and ${ }^{5}$ Institute of Pharmaceutical Sciences, Swiss Federal Institute of Technology Zurich, CH-8090 Zurich, Switzerland
}

Gastrin-releasing peptide (GRP) is a spinal itch transmitter expressed by a small population of dorsal horn interneurons (GRP neurons). The contribution of these neurons to spinal itch relay is still only incompletely understood, and their potential contribution to painrelated behaviors remains controversial. Here, we have addressed this question in a series of experiments performed in GRP::cre and GRP::eGFP transgenic male mice. We combined behavioral tests with neuronal circuit tracing, morphology, chemogenetics, optogenetics, and electrophysiology to obtain a more comprehensive picture. We found that GRP neurons form a rather homogeneous population of central cell-like excitatory neurons located in lamina II of the superficial dorsal horn. Multicolor high-resolution confocal microscopy and optogenetic experiments demonstrated that GRP neurons receive direct input from MrgprA3-positive pruritoceptors. Anterograde HSV-based neuronal tracing initiated from GRP neurons revealed ascending polysynaptic projections to distinct areas and nuclei in the brainstem, midbrain, thalamus, and the somatosensory cortex. Spinally restricted ablation of GRP neurons reduced itch-related behaviors to different pruritogens, whereas their chemogenetic excitation elicited itch-like behaviors and facilitated responses to several pruritogens. By contrast, responses to painful stimuli remained unaltered. These data confirm a critical role of dorsal horn GRP neurons in spinal itch transmission but do not support a role in pain.

Key words: chemogenetics; interneuron; neuronal tracing; nociception; optogenetics; pruritus

\section{Significance Statement}

Dorsal horn gastrin-releasing peptide neurons serve a well-established function in the spinal transmission of pruritic (itch) signals. A potential role in the transmission of nociceptive (pain) signals has remained controversial. Our results provide further support for a critical role of dorsal horn gastrin-releasing peptide neurons in itch circuits, but we failed to find evidence supporting a role in pain.

\section{Introduction}

Acute itch, very much like pain, is a protective sense that makes us aware of potentially harmful stimuli or events. Activation of pru-

Received Oct. 3, 2018; revised Jan. 10, 2019; accepted Jan. 10, 2019.

Author contributions: G.W.A. wrote the first draft of the paper; M.P., E.P., H.C.J., H.W., and H.U.Z. edited the paper; G.W.A., E.P., and H.U.Z. designed research; G.W.A., M.P., E.P., and L.H. performed research; H.W. contributed unpublished reagents/analytic tools; G.W.A., M.P., E.P., J.-M.F., and H.W. analyzed data; H.U.Z. wrote the manuscript.

The work has been supported by Swiss National Science Foundation Grant 176398, European Research Council Advanced Investigator Grant AdvG 250128, and a Wellcome Trust Collaborative Award in Science (200183/Z/15/Z) to H.U.Z., and Olga Mayenfisch Stiftung grant to H.W. We thank Xinzhong Dong (Johns Hopkins University) for the MrgrpA3:.:cre mice; Carmen Birchmeier (Max-Delbrück Center for Molecular Medicine, Berlin-Buch) for providing the Lmx1b and TIx3 antibodies; Martin Schmelz (University of Heidelberg) for helpful input on the manuscript; and Isabelle Kellenberger for genotyping of transgenic mice.

The authors declare no competing financial interests.

Correspondence should be addressed to Hanns Ulrich Zeilhofer at zeilhofer@pharma.uzh.ch.

https://doi.org/10.1523/JNEUROSCI.2559-18.2019

Copyright $\odot 2019$ the authors $\quad 0270-6474 / 19 / 392238-13 \$ 15.00 / 0$ ritoceptive sensory afferents ("itch fibers") elicits the desire to scratch aiming at the removal of irritant stimuli from the skin. Similar to sensory neurons activated by painful stimuli, pruritoceptors have their cell bodies in the DRGs and trigeminal ganglia, from where they send peripheral axons to the skin and central axons to the spinal cord or brainstem. The termination areas of the central axons lie in the superficial layers of the spinal dorsal horn or in the brainstem, where they make excitatory synaptic connections with local second-order neurons.

In the last decade, several key players (receptors and neurons) of peripheral and spinal itch pathways have been identified. In the periphery, members of the mas-related GPCR family have been found to play important roles as receptors for pruritogens. MrgprA3 (MrgprX1 in humans) underlies the pruritic actions of chloroquine, whereas other members of the Mrgpr family (A1, $\mathrm{A} 4$, and C11) respond to the pruritic peptides FMRF, NPFF, and BAM 8-22 (Liu et al., 2009). MrgprA3-positive pruritoceptors 
are activated by a variety of itch stimuli, including activators of histaminergic and nonhistaminergic itch, and their ablation strongly reduces itch responses (Han et al., 2013). Additional subsets of peripheral pruritoceptors are characterized by the expression of brain-type natriuretic peptide (Nppb) (Usoskin et al., 2015) or somatostatin (Huang et al., 2018).

At the spinal level, a critical role in itch has been found for the receptor activated by the neuropeptide gastrin-releasing peptide, the gastrin-releasing peptide (GRP) receptor (GRPR) (Y.G. Sun and Chen, 2007). Mice lacking GRPR display reduced scratching responses to chloroquine, compound $48 / 80$, and activation of the protease activated receptor 2 (PAR2). The respective neurons that express the GRPR (GRPR neurons) are located in the superficial layers of the dorsal horn (Y.G. Sun and Chen, 2007). Recent work has shown that these neurons are local spinal interneurons that excite spinoparabrachial projection neurons to relay pruritoceptive signals to supraspinal CNS areas (Mu et al., 2017). Ablation of the GRPR neurons almost completely abolishes itch-related behaviors evoked by different pruritogens, including histamine, chloroquine, and serotonin (Y.G. Sun et al., 2009).

The studies on mice lacking the GRP receptor (Y.G. Sun and Chen, 2007) or the GRPR neurons (Y.G. Sun et al., 2009) suggest a specific role not only of GRP but also of the GRP releasing neurons in itch, consistent with a "labeled line" for itch that extends from the periphery at least to the spinal cord. However, a more recent study (S. Sun et al., 2017) that used selective activation and ablation of GRP-expressing neurons reported evidence for an additional role of these neurons in the transmission of low-intensity pain signals. Here, we have readdressed this question and undertook an in-depth analysis of the integration of GRP neurons in dorsal horn neuronal circuits and of their contributions to itch and pain-related behaviors. Our new data are consistent with a critical and rather specific role of these neurons in the spinal processing of pruritoceptive signals.

\section{Materials and Methods}

Mice. Experiments were performed on 6- to 12-week-old male mice kept at a 12:12 h light/dark cycle. Permissions have been obtained from the Canton of Zurich (permissions 257/2014, 031/2016, 023/2016, and 063/ 2016). For details on the genetically modified mice used in this study, see Table 1.

Immunohistochemistry and image analysis. Mice were perfused transcardially with $20 \mathrm{ml}$ of ice-cold ACSF $(125 \mathrm{NaCl}, 2.5 \mathrm{KCl}, 1.25$ $\mathrm{NaH}_{2} \mathrm{PO}_{4}, 25 \mathrm{NaHCO}_{3}, 1 \mathrm{MgCl}_{2}, 2 \mathrm{CaCl}_{2}, 20$ glucose equilibrated with $95 \% \mathrm{O}_{2} / 5 \% \mathrm{CO}_{2}$ ) followed by $100 \mathrm{ml}$ of $4 \%$ ice-cold PFA (in $0.1 \mathrm{M}$ sodium phosphate buffer, $\mathrm{pH}$ 7.4). Lumbar spinal cords and brains were dissected and postfixed for 2 and $4 \mathrm{~h}$, respectively, with 4\% PFA on ice. The postfixed tissue was briefly washed with $0.1 \mathrm{M}$ sodium phosphate buffer and then incubated for cryoprotection in $25 \%$ sucrose solution (in $0.1 \mathrm{~m}$ sodium phosphate buffer) overnight at $4^{\circ} \mathrm{C}$. Tissue was embedded in NEG50 frozen section medium (Richard-Allen Scientific) and stored at $-80^{\circ} \mathrm{C}$ until use. Spinal tissue and DRG tissue were cut into 30 and 16 $\mu \mathrm{m}$ cryosections, respectively, using Hyrax C60 cryostat (Carl Zeiss) and mounted onto Superfrost Plus microscope slides (Thermo Fisher Scientific). Brain tissue and spinal cord tissue used for the neurochemical characterization of GRP-eGFP cells were cut into $40 \mu \mathrm{m}$ section with Hyrax KS 34 microtome (Carl Zeiss) and transferred to PBS for immunostaining on free-floating sections. Immunofluorescence staining was performed using combinations of antibodies and fluorescent conjugates listed in Table 1. Spinal cord and brain sections were incubated with primary antibodies in PBS supplemented with $0.3 \%$ Triton X-100 and $10 \%$ normal donkey serum overnight at $4^{\circ} \mathrm{C}$ and with secondary antibodies solution in PBS supplemented with $0.3 \%$ Triton X-100 for $1 \mathrm{~h}$ at room temperature. Sections were washed 3 times for 5-10 min in PBS after each antibody incubation. Images were taken using an LSM 710
Table 1. Resource table

\begin{tabular}{|c|c|c|}
\hline Reagent & Resource & Identifier \\
\hline \multicolumn{3}{|l|}{ Mice } \\
\hline GRP::cre & $\begin{array}{l}\text { Mutant Mouse Regional } \\
\text { Resource Centers }\end{array}$ & RRID:MMRRC_031182-UCD \\
\hline GRP::.eGFP & $\begin{array}{l}\text { Mutant Mouse Regional } \\
\text { Resource Centers }\end{array}$ & RRID:MMRRC_010444-UCD \\
\hline MrgrpA3::.cre & Xinzhong Dong & Han et al., 2013 \\
\hline ROSA26 $6^{\mid s l-C h R 2}$ & The Jackson Laboratory & RRID:IMSR_JAX:012569 \\
\hline ROSA $A^{I S-I D T R}$ & The Jackson Laboratory & RRID:IMSR_JAX:007900 \\
\hline ROSA $A^{\text {Isl-tdTom }}$ & The Jackson Laboratory & RRID:IMSR_JAX:007914 \\
\hline ROSA $A^{S L-T V A}$ & Dieter Saur & Seidler B et al., 2008 \\
\hline
\end{tabular}

Antibodies (dilution)

Chicken anti-GFP (1:1000)

Mouse anti-calbindin (1:5000)

Goat anti- Pax2 (1:200)

Goat anti-CGRP (1:500)

Goat anti-tdTomato (1:1000)

Guinea pig anti-Lmx1b $(1: 10,000)$

Guinea pig anti-VGluT2 $(1: 10,000)$

Rabbit anti-GFP (1:1000)

Rabbit anti-NeuN (1:3000)

Rabbit anti-PKC $\gamma(1: 1000)$

Rabbit anti-TIx3 (1:10,000)

Rabbit anti-Homer1 (1:2000)

Rat anti-mCherry (1:1000)

AlexaFluor-488-conkey anti-chicken $(1: 800)$

AlexaFluor-488-donkey anti-goat (1:800)

AlexaFluor-488-donkey anti-rabbit (1:800)

AlexaFluor-647-donkey anti-goat (1:800)

AlexaFluor-647-donkey anti-guinea pig (1:800)

AlexaFluor-647-donkey anti-rabbit (1:800)

Cy3-donkey anti-goat (1:800)

Cy3-donkey anti-mouse (1:800)

Cy3-donkey anti-rabbit (1:800)

Cy3-donkey anti-rat (1:800)

DyLight 549-donkey anti-guinea pig (1:800)

Biotin-donkey anti-rabbit (1:300)

Fluorescent conjugates (dilution)

Pacific Blue-Streptavidin (1:1000)

AlexaFluor-647-Isolectin GS-IB4

(1:500)

RNAscope ISH probes

Mm-Grp

Mm-Grp-C2

Mm-Grpr-C2

tdTomato

Viruses

AAV1.hSyn.flex.hM3Dq-mCherry

SAD.Rabies $\Delta$ G.eGFP (EnvA)

H129 $\Delta$ TK-TT

\begin{tabular}{|c|c|}
\hline Thermo Fisher Scientific & RRID:AB_2534023 \\
\hline Swant & RRID:AB_10000347 \\
\hline R\&D Systems & RRID:AB_10889828 \\
\hline Abcam & RRID:AB_725807 \\
\hline SICGEN & RRID:AB_2722750 \\
\hline Carmen Birchmeier & Muller et al. 2002 \\
\hline Millipore & RRID:AB_2665454 \\
\hline Molecular Probes & RRID:AB_221570 \\
\hline Abcam & RRID:AB_10711153 \\
\hline Santa Cruz Biotechnology & RRID:AB_632234 \\
\hline Carmen Birchmeier & Muller et al. 2002 \\
\hline Synaptic Systems & RRID:AB_2120990 \\
\hline Thermo Fisher Scientific & RRID:AB_2536611 \\
\hline $\begin{array}{l}\text { Jackson ImmunoResearch } \\
\text { Laboratories }\end{array}$ & RRID:AB_2340376 \\
\hline $\begin{array}{l}\text { Jackson ImmunoResearch } \\
\text { Laboratories }\end{array}$ & RRID:AB_2340430 \\
\hline $\begin{array}{l}\text { Jackson ImmunoResearch } \\
\text { Laboratories }\end{array}$ & RRID:AB_2340619 \\
\hline $\begin{array}{l}\text { Jackson ImmunoResearch } \\
\text { Laboratories }\end{array}$ & RRID:AB_2340437 \\
\hline $\begin{array}{l}\text { Jackson ImmunoResearch } \\
\text { Laboratories }\end{array}$ & RRID:AB_2340477 \\
\hline $\begin{array}{l}\text { Jackson ImmunoResearch } \\
\text { Laboratories }\end{array}$ & RRID:AB_2340626 \\
\hline $\begin{array}{l}\text { Jackson ImmunoResearch } \\
\text { Laboratories }\end{array}$ & RRID:AB_2340413 \\
\hline $\begin{array}{l}\text { Jackson ImmunoResearch } \\
\text { Laboratories }\end{array}$ & RRID:AB_2315777 \\
\hline $\begin{array}{l}\text { Jackson ImmunoResearch } \\
\text { Laboratories }\end{array}$ & RRID:AB_2307443 \\
\hline $\begin{array}{l}\text { Jackson ImmunoResearch } \\
\text { Laboratories }\end{array}$ & RRID:AB_2340667 \\
\hline $\begin{array}{l}\text { Jackson ImmunoResearch } \\
\text { Laboratories }\end{array}$ & Catalog \#706-506-148 \\
\hline Thermo Fisher Scientific & RRID:AB_228212 \\
\hline Thermo Fisher Scientific & Catalog \#S11222 \\
\hline Thermo Fisher Scientific & RRID:SCR_014365 \\
\hline Advanced Cell Diagnostics & Catalog \#317861 \\
\hline Advanced Cell Diagnostics & Catalog \#317861-C2 \\
\hline Advanced Cell Diagnostics & Catalog \#317871-C2 \\
\hline Advanced Cell Diagnostics & Catalog \#317041 \\
\hline Penn Vector Core & Foster et al., 2015 \\
\hline Custom production & Albisetti et al., 2017 \\
\hline Enquist Lynn, CNNV & Lo and Anderson, 2011 \\
\hline
\end{tabular}

confocal laser scanning microscope (Carl Zeiss) equipped with a 0.8 NA $\times 20$ Plan-apochromat objective and a 1.3 NA $\times 40$ EC PlanNeofluar oil-immersion objective. Maximum intensity projections were created from $Z$-stack images (3-15 optical sections, $0.7-1.5 \mu \mathrm{m}$ step size) and processed using ImageJ software (National Institutes of Health).

Immunofluorescence images of brain sections and chromogenic ISH images of spinal cord tissue were taken using an Axio Imager Z1 microscope (Carl Zeiss) equipped with $0.3 \mathrm{NA} \times 10 \mathrm{EC}$ Plan-Neofluar and 0.8 
NA $\times 20$ Plan-apochromat objectives. To quantify the number of cells, at least 3 animals and three sections per animal were analyzed. Cell counting was performed using the ImageJ Cell Counter plug-in.

Dendritic morphology of GRP neurons was studied using 3D imaging of cleared lumbar spinal tissue. For tissue clearing, a modified passive CLARITY (Chung and Deisseroth, 2013) protocol was used. In brief, GRP::cre mice crossed to the ROSA26 $6^{\text {lsl-TVA }}$ reporter were intraspinally injected with EnvA-pseudotyped rabies virus (SAD.Rabies $\Delta$ G.eGFP (EnvA)) and perfused with 4\% PFA $5 \mathrm{~d}$ after virus injection. Lumbar spinal tissue dissected and postfixed overnight in $4 \%$ PFA at $4^{\circ} \mathrm{C}$ followed by a subsequent overnight incubation at $4^{\circ} \mathrm{C}$ in a PBS solution containing $4 \%$ acrylamide (Bio-Rad) and 0.25\% VA-044 (Novachem). Spinal cord tissue was then incubated for $3 \mathrm{~h}$ at $37^{\circ} \mathrm{C}$ for acrylamide polymerization and incubated overnight in clearing solution as follows: $200 \mathrm{~mm}$ SDS (Sigma-Aldrich), $200 \mathrm{~mm}$ boric acid (Sigma-Aldrich), $\mathrm{pH} 8.5$, at $37^{\circ} \mathrm{C}$ on a shaker at $180 \mathrm{rpm}$. Spinal tissue was washed three times in PBS, incubated in $88 \%$ Histodenz (Sigma-Aldrich) solution in PBS (refractory index adjusted to 1.457) overnight, and mounted in the same solution. Imaging was performed on a TCS SP8 upright multiphoton microscope equipped with NA $1.025 \times$ water objective, HyD-RLD detectors (Leica Microsystems) and InSigth DS Dual (Spectraphysics) NIR laser tuned to $950 \mathrm{~nm}$ for eGFP excitation. Data were segmented using Imaris version 8 (Bitplane), and single neurons were manually selected for the size calculations and manual classification. In total, 32 cells from two mice were analyzed.

In situ hybridization. Spinal tissue used for ISH was dissected from 6- to 12-week-old mice, collected in $1.5 \mathrm{ml}$ Eppendorf tubes, and immediately frozen in liquid nitrogen. Tissue was embedded in NEG50 frozen section medium (Richard-Allen Scientific), cut into 20 $\mu \mathrm{m}$ sections, and hybridized using DIGlabeled riboprobes (overnight at $70^{\circ} \mathrm{C}$ ) or with the probes designed for RNAscope Fluorescent Multiplex ISH listed in Table 1. Tissue hybridized using DIG-labeled riboprobes was incubated with sheep anti-DIG-alkaline-phosphatase-conjugated antibody (1:1500, Roche Diagnostics) and developed in NTMT solution (0.1 M Tris, $\mathrm{pH} 9.5,0.1 \mathrm{M} \mathrm{NaCl}, 50 \mathrm{~mm} \mathrm{MgCl}_{2}, 0.1 \%$ Tween 20) containing $0.035 \mathrm{mg} / \mathrm{ml}$ NBT (AppliChem) and $0.017 \mathrm{mg} / \mathrm{ml} \mathrm{BCIP} \mathrm{(AppliChem)} \mathrm{for} 3 \mathrm{~d}$.

Intrathecal injections of diphtheria toxin (DTX). Mice were anesthetized with isoflurane, and a small skin incision was performed on the back of the animal; $3 \mu \mathrm{l}$ of $5 \mathrm{ng} / \mu \mathrm{l}$ DTX suspension (in filtered $0.9 \% \mathrm{NaCl}, 8 \%$ dextrose) was injected through the intervertebral space L1/L2 using a $10 \mu \mathrm{l}$ Hamilton syringe with a needle adapter to limit the injection depth to $2.5 \mathrm{~mm}$.

Intraspinal virus injections. Intraspinal virus injections were made in isoflurane-anesthe-

tized mice using a motorized stereotaxic frame (David Kopf Instruments and Neurostar). Three separate unilateral injections of $300 \mathrm{nl}$ each were performed at a depth of $300 \mu \mathrm{m}$ using glass micropipettes connected to a PHD ultra nanomite syringe pump (Harvard Apparatus) as described previously (Albisetti et al., 2017; Haenraets et al., 2018). The following viruses were used: for chemogenetic activation, AAV1.hSyn.flex. hM3Dq-mCherry $\left(6.5 \times 10^{12} \mathrm{IU} / \mathrm{ml}\right)$; for dendritic morphology analy-
B

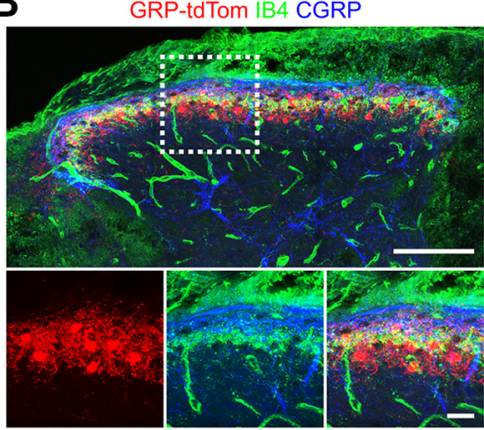

D

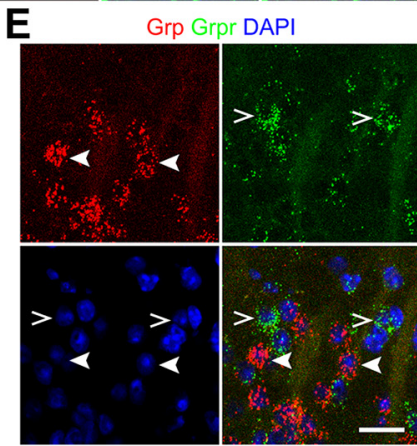

H

G
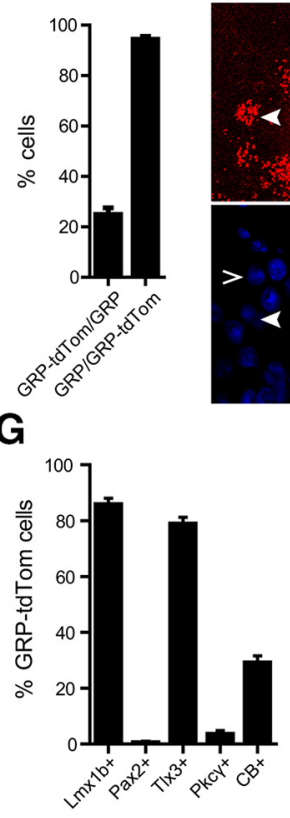

GRP-tdTom NeuN

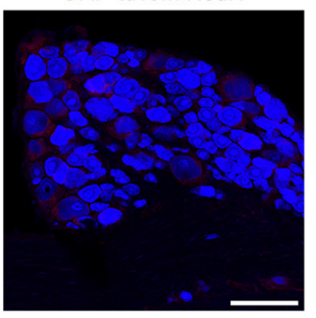

Figure 1. Characterization of GRP-cre-expressing neurons. $\boldsymbol{A}$, Immunofluorescence staining on a transversal section of lumbar Quantification of C.E, Double ISH shows that Grp-and Grpr-expressing neurons are two nonoverlapping neuronal populations. Full that GRP-tdTom neurons express the excitatory neuronal markers Lmx1b but not the inhibitory marker Pax2. G, Quantitative colocalization studied on spinal sections of GRP::Cre; ROSA26 $6^{\text {Isl-tdTom }}$ mice stained with antibodies to Lmx1b, Pax2, TIx3, PKC $\gamma$, and

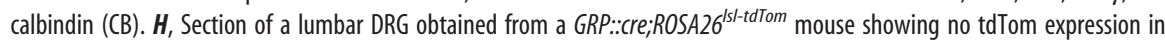
primary sensory neurons. Scale bars $100 \mu \mathrm{m}$ in overviews and $20 \mu \mathrm{m}$ in higher magnifications $(\boldsymbol{A}, \boldsymbol{B}, \boldsymbol{C}, \boldsymbol{E}, \boldsymbol{F})$, and $100 \mu \mathrm{m}(\boldsymbol{H})$.

ses, SAD.Rabies $\Delta$ G.eGFP (EnvA, $1.5 \times 10^{7} \mathrm{IU} / \mathrm{ml}$ ); and for anterograde tracing, H129DTK-TT. For the latter replication competent virus, no titer was available. For details on the viruses, see Table 1.

Behavioral analyses. All behavioral tests were performed during light phase. The experimenter was blinded for the genotype of the mice. For nociception assays, the mice were placed into Plexiglas boxes and allowed to adapt to the new environment for $30 \mathrm{~min}$. In pharmacogenetic experiments, behavioral pain tests were performed 1-2 weeks after surgery, from 1.5 to $2.5 \mathrm{~h}$ after intraperitoneal clozapine $N$-oxide (CNO, Enzo Life Sciences) injection ( $2 \mathrm{mg} / \mathrm{kg}$ ). Mechanical withdrawal thresholds were assessed using an electronic von Frey anesthesiometer (IITC), and the maximal force applied on the mouse paw was restricted to $6 \mathrm{~g}$. Withdrawal latencies to noxious heat were assessed using Hargreaves test apparatus (IITC) with a temperature-controlled glass platform set to $30^{\circ} \mathrm{C}$. Withdrawal latencies to noxious cold were assessed cooling the 5-mm-thick borosilicate glass platform directly under the mouse hindpaw using a cold probe (powdered dry ice compressed into a $1 \mathrm{~cm}$ large syringe) (Brenner et al., 2012). For Hargreaves and cold tests, cutoff times were set to 32 and 24 s, respectively. For von Frey, Hargreaves, and cold 
A

cell 1
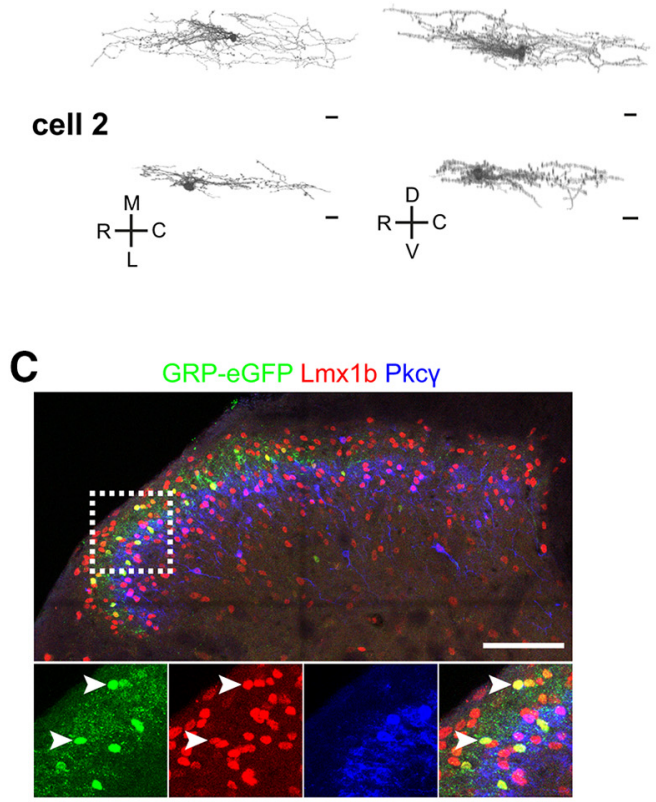

E

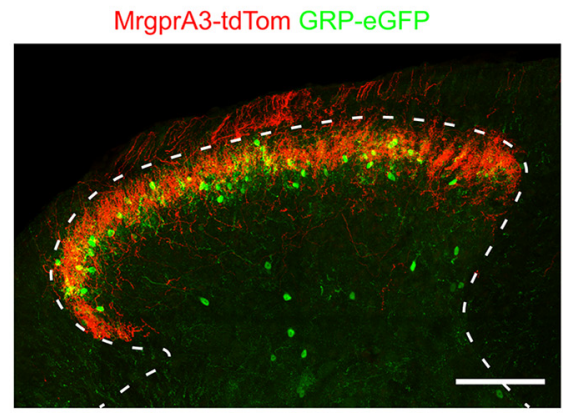

G

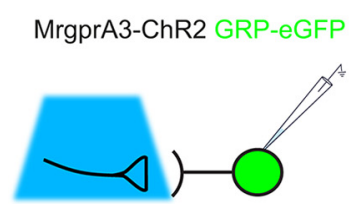

B

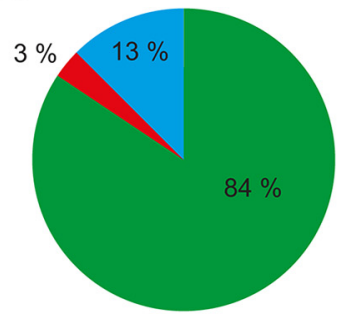

central unclassified $\square$ vertical

D

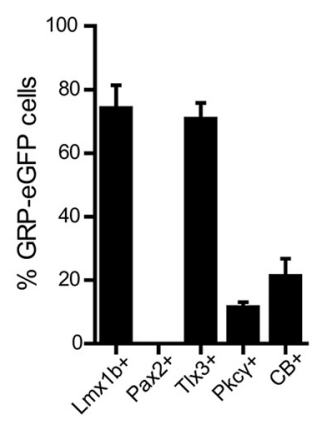

F MrgprA3-tdTom GRP-eGFP vGluT2 HOMER

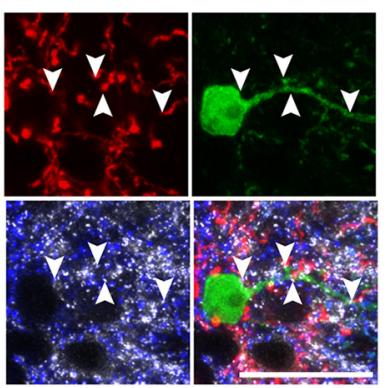

1
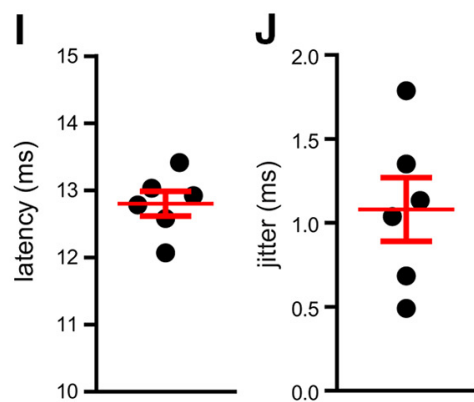

Figure 2. Morphology and MrgprA3 ${ }^{+}$primary sensory input of GRP-cre-expressing neurons. $A$, Examples of two GRP central neurons form a spinal cord of GRP:::cre ROSA26 ${ }^{I s-T V A}$ mice infected with SAD.Rabies $\triangle$ G.eGFP (EnvA) virus. The morphology of the same cells is shown in the dorsoventral (left) and mediolateral view (right). Scale bars, $20 \mu \mathrm{m}$. B, Quantification of the morphological analysis of GRP neurons. C, Immunofluorescence staining of a lumbar spinal cord section of GRP::eGFP mice showing GRP-eGFP colocalization with the excitatory marker Lmx $1 \mathrm{~b}$ in lamina II. Arrowheads indicate examples of colocalization of GRPeGFP and Lmx1b immunoreactivity. Scale bar, $100 \mu \mathrm{m}$. D, Quantitative analysis verified that GRP-eGFP neurons and GRP-cre neurons exhibit similar neurochemical characteristics (compare Fig. 1G). In bar charts, data are mean \pm SEM. E, Overview of the

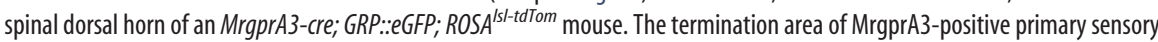
neurons largely overlaps with the location where the GRP-eGFP cells are located. Scale bar, $100 \mu \mathrm{m}$. F, High-magnification images of a GRP-eGFP cell receiving direct synaptic input from MrgprA3-positive fibers. Arrowheads indicate MrgprA3-positive excitatory synaptic contacts onto a GRP-eGFP cell. Scale bar, $20 \mu \mathrm{m}$. G, Schematic showing optogenetic activation of MrgprA3-expressing primary afferent terminals (MrgprA3-ChR2) and targeted recordings from eGFP-positive GRP neurons (GRP-eGFP). H, Superposition of 20 consecutive light-evoked EPSCs traces recorded from GRP-eGFP neurons. Blue area represents $473 \mathrm{~nm}, 4 \mathrm{~ms}, 0.1 \mathrm{~Hz}$. Gray represents individual responses. Black represents average response. $I, J$, Dot plots illustrate latency $(\boldsymbol{I})$ and jitter $(\boldsymbol{J})$ of light-elicited EPSCs recorded from GRP-eGFP cells ( $n=6$, from 5 animals). Circles represent individual cells. Error bars indicate SEM.

tests, eight measurements were taken per hindpaw in 5-10 min intervals, and average hindpaw withdrawal thresholds were calculated for each mouse. Motor coordination was tested using a rotarod instrument (IITC) that linearly accelerated from 0 to $40 \mathrm{rpm}$ over $5 \mathrm{~min}$, and the latency to fall was measured for each mouse. Two training sessions were performed before the test experiment. Six measurements were taken for each mouse. For pinprick test and brush tests, measurements were taken by stimulating the plantar surface of the mouse hindpaw with a blunted G26 needle and a soft paint brush, respectively. Ten measurements were taken at the interval of $2 \mathrm{~min}$, and responses were scored as " 0 " for no reaction or " 1 " if the animal responded. For behavioral experiments on itch, mice were placed into Plexiglas cylinders for $30 \mathrm{~min}$ of adaptation. For intradermal injection of pruritogens, the mice were restrained without anesthesia, and $10 \mu \mathrm{l}$ of pruritogen was injected intracutaneously into the skin above the calf using a 30 gauge needle. The injection site was shaved $1 \mathrm{~d}$ before the experiment. The following pruritogens (dissolved in $0.9 \%$ filtered saline) were used: chloroquine diphosphate salt $(8 \mu \mathrm{g} / \mu \mathrm{l}$, SigmaAldrich), histamine $(10 \mu \mathrm{g} / \mu \mathrm{l}$, Sigma-Aldrich), $\alpha$-methyl serotonin maleate salt $(2 \mu \mathrm{g} / \mu \mathrm{l}$, Sigma-Aldrich), and SLIGRL-NH2 $(6.57 \mu \mathrm{g} /$ $\mu \mathrm{l}, \mathrm{Abcam})$. The mice were videotaped for 30 min after the injection of the pruritogen, and the biting time directed toward the injected site was quantified. In pharmacogenetic experiments, mice were returned for $1 \mathrm{~h}$ to their home cage immediately after intraperitoneal CNO injection $(2 \mathrm{mg} / \mathrm{kg})$. Mice were then allowed to adapt for $15 \mathrm{~min}$ and then videotaped for $45 \mathrm{~min}$ to assess $\mathrm{CNO}$ induced itch behavior. Chloroquine diphosphate salt $(8 \mu \mathrm{g} / \mu \mathrm{l})$ and histamine $(10 \mu \mathrm{g} /$ $\mu \mathrm{l})$ were injected intracutaneously $2 \mathrm{~h}$ after CNO injection, and mice were videotaped for $30 \mathrm{~min}$ immediately after pruritogen administration. All the recordings were performed in the absence of the experimenter, and biting time was analyzed offline. In pharmacogenetics experiments that tested the dose dependence of $\mathrm{CNO}(0.7 \mathrm{mg} / \mathrm{kg}, 0.1$ $\mathrm{mg} / \mathrm{kg}, 0.02 \mathrm{mg} / \mathrm{kg}$ ), and in all nociception and itch assays, data acquisition started 75 min after $\mathrm{CNO}$ administration.

Electrophysiology and optogenetics. Transverse $400-\mu \mathrm{m}$-thick spinal cord slices were prepared from 4- to 5-week-old mice as described previously (Dugué et al., 2009; Punnakkal et al., 2014). Whole-cell patch-clamp experiments were made at room temperature using an EPC 9 amplifier (HEKA Elektronik) controlled with Patchmaster acquisition software. GRP-eGFP-positive neurons were identified by their green fluorescence in slices prepared from MrgprA3::cre;GRP::eGFP;ROSA26 $6^{\text {lsl-ChR2 }}$ triple transgenic mice. During recordings, slices were continuously superfused at the rate of $1-2 \mathrm{ml} / \mathrm{min}$ with ACSF containing the following (in mM): $120 \mathrm{NaCl}, 2.5 \mathrm{KCl}, 1.25$ $\mathrm{NaH}_{2} \mathrm{PO}_{4}, 26 \mathrm{NaHCO}_{3}, 5$ HEPES, $1 \mathrm{MgCl}_{2}, 2$ $\mathrm{CaCl}_{2}$, and 14.6 glucose, $\mathrm{pH} 7.4$, equilibrated 
with $95 \% \mathrm{O}_{2} / 5 \% \mathrm{CO}_{2}$. Patch pipettes (borosilicate glass; 3.5-4.5 $\mathrm{M} \Omega$ ) were filled with intracellular solution containing the following (in $\mathrm{mM}$ ): 130 potassium gluconate, $5 \mathrm{NaCl}, 1$ EGTA, 10 HEPES, 5 Mg-ATP, 0.5 Na-GTP, pH 7.35 (290-300 mosm/L). Membrane potentials were corrected for a liquid junction potential of $15.2 \mathrm{mV}$. Access resistance of each neuron was continuously monitored with short hyperpolarizing voltage steps. Optogenetic stimulation of MrgprA3-positive terminals was done with field illumination through a $40 \times$ water-immersion objective using a Polychrome V monochromator (TILL Photonics). MrgprA3-positive terminals were stimulated with brief, 4-ms-long pulses of blue light ( $473 \pm 5 \mathrm{~nm}$ wave lengths, $1.15 \mathrm{~mW}$ ). Average failure rates $<5 \%$ (Doyle and Andresen, 2001) and jitters (intraneuronal SD of EPSC latency) $\leq 1.6$ ms (Wang and Zylka, 2009) were used as criteria to classify light-evoked EPSCs as monosynaptic. The latency was calculated between the onset of light commands and the onset of EPSCs. Electronic and mechanical delay of the system was calculated to be $3.38 \mathrm{~ms}$ and was subtracted from the latency values. Light-evoked EPSCs were recorded at holding potential of $-70 \mathrm{mV}$

Experimental design and statistical tests. All experiments were designed to allow comparisons between exactly two groups. Statistical comparisons were made using unpaired $t$ tests (GraphPad Prism version 5 for Windows, GraphPad Software). All data are given as mean \pm SEM. Results of statistical tests are given as the $t$ score $(t)$ and the $\alpha$ error $(p)$. $p$ values $\leq 0.05$ were considered statistically significant. Numbers of experiments (cells or mice) are provided in Results and in the figure legends. All experiments were done in male mice. All numerical data are available as Excel files, including the data that support the findings of this study (www.G-Node.org; identifier doi: 10.12751/g-node.50baa6).

\section{Results}

To examine the integration of GRP neurons in dorsal horn neuronal circuits and to investigate their potential role in itch and pain-related behaviors, we used GRP::cre BAC transgenic mice. We first characterized the spinal expression pattern of the GRP:: cre transgene and crossed GRP::cre mice to cre-dependent tdTomato (Ai14 tdTom) reporter mice. Lumbar spinal cord sections prepared from adult (6- to 10 -week-old mice) double transgenic offspring revealed that GRP-tdTom-positive neurons were restricted to lamina II of the superficial dorsal horn. Most GRPtdTom-positive neurons were located ventral to the calcitonin gene-related peptide (CGRP)-positive layer and dorsal to the $\mathrm{PKC} \gamma$ neuron layer partially overlapping with the termination area of IB4-positive sensory nerve fibers (Fig. $1 A, B$ ). Double ISH experiments (7 sections from 2 mice) verified that GRP-tdTom was only present in Grp mRNA-positive neurons (94.4 $\pm 1.5 \%$ ), but only $24.9 \pm 2.6 \%$ of neurons with Grp ISH signals were also tdTom-positive (Fig. 1C,D). Double ISH experiments using probes for Grp and its receptor, the Grpr, demonstrated in addition that Grp and Grpr marked distinct dorsal horn neuron populations (Fig. 1E). Subsequent neurochemical characterization of spinal GRP-tdTom neurons (8-14 sections from 3-5 mice were used to quantify GRP-tdTom colocalization with each of the neurochemical marker) showed that $85.8 \pm 2.2 \%$ and $78.8 \pm 2.4 \%$ of these neurons coexpressed, respectively, the excitatory interneuron markers Lmx1b and Tlx3, whereas virtually no overlap $(0.5 \pm$ $0.5 \%$ ) with the inhibitory interneuron marker Pax2 was found (Fig. $1 F, G) ; 29.1 \pm 2.5 \%$ of GRP-tdTom neurons expressed calbindin, and only $3.5 \pm 1.4 \%$ expressed PKC $\gamma$ (Fig. $1 G$ ). No GRPtdTom was detected in the DRGs (16 sections from 4 mice) (Fig. $1 H$ )

We next investigated the dendritic morphology of dorsal horn GRP neurons ( 32 cells from 2 mice). The combination of sparse neuronal labeling and large-volume imaging allowed the characterization of dendritic tree architecture in three dimensions. The sparse labeling was achieved by injecting

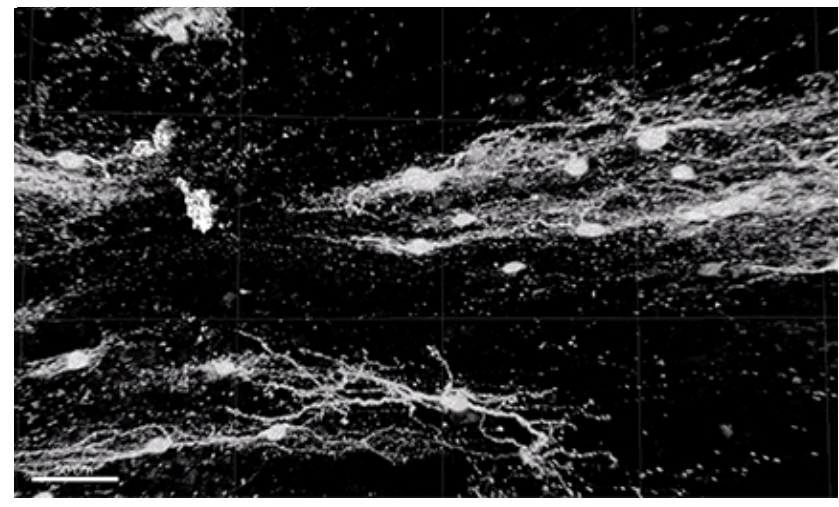

Movie 1. Dendritic morphology of dorsal horn GRP-cre neurons visualized using the CLARITY technique in a whole mount of the lumbar spinal cord of a GRP:.:cre mouse injected with SAD.Rabies $\Delta$ G.eGFP virus.

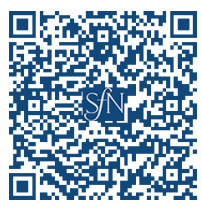

lumbar spinal cords of the GRP::cre; ROSA26 $6^{\text {lsl-TVA }}$ double transgenic mice with a glycoprotein-deficient eGFP reporter rabies virus (SAD.Rabies $\Delta$ G.eGFP (EnvA)). Because no transcomplementation of the glycoprotein-deficient rabies virus was done, secondary infection did not occur and only few GRP-cre neurons expressed eGFP. The lumbar spinal tissue was then cleared using modified passive CLARITY, and eGFP fluorescence was visualized in the whole mounts with 2-photon microscopy technique. Tissue volumes of $1.0 \mathrm{~mm} \times 0.5 \mathrm{~mm} \times 0.6 \mathrm{~mm}$ (rostrocaudal $\times$ mediolateral $\times$ dorsoventral) size were imaged. Because of the sparse labeling, it was possible to segment individual cells from the visualized volumes and assess the full dendritic arbor architecture in $3 \mathrm{D}$. The vast majority of labeled neurons displayed a central cell-like morphology (Fig. 2A, B; Movie 1).

Results from several behavioral studies indicate that GRP and its receptor the GRPR play a critical role in itch and suggest that GRP-expressing neurons are part of an itch processing circuit in the spinal dorsal horn (Y.G. Sun and Chen, 2007; Y.G. Sun et al., 2009; S. Sun et al., 2017). To investigate whether GRP neurons receive synaptic input from MrgprA3-positive pruritoceptive sensory fibers, we used GRP::eGFP transgenic mice. Immunofluorescence analyses (7-9 sections from 3 mice were used to quantify GRP-eGFP colocalization with each of the neurochemical marker) verified that GRP-eGFP neurons exhibited neurochemical features that were very similar to the one of the GRP-cre neurons characterized above $(73.9 \pm 7.5 \%$ colocalization with Lmx1b, $70.5 \pm 5.3 \%$ with Tlx3, $11.2 \pm 1.8 \%$ with $\mathrm{PKC} \gamma$, $21.0 \pm 5.7 \%$ with calbindin and no overlap with Pax2) (Fig. $2 C, D$; see also Fig. $1 F, G$ ). We next prepared lumbar spinal cord sections from MrgprA3::cre; GRP::eGFP; ROSA26 $6^{\text {lsl-tdTom }}$ triple transgenic mice. These sections revealed that MrgprA3-positive pruritoceptors terminate mainly in lamina II (i.e., in the same lamina where most GRP-eGFP neurons are located) (Fig. 2E). Staining of sections from these mice with antiserum against HOMER, a postsynaptic marker of glutamatergic synapses (Gutierrez-Mecinas et al., 2016b), and antiserum against vGluT2 indicates that MrgprA3-positive fibers form synaptic contacts with GRP-eGFP neurons in lamina II of the dorsal horn (Fig. $2 F$ ). To functionally demonstrate the presence of synaptic connections, we prepared transverse slices of the lumbar spinal cord from MrgprA3::cre; GRP::eGFP; ROSA26 $6^{\text {sl-ChR2 }}$ triple transgenic mice. In these slices, we performed whole-cell recordings from 
A
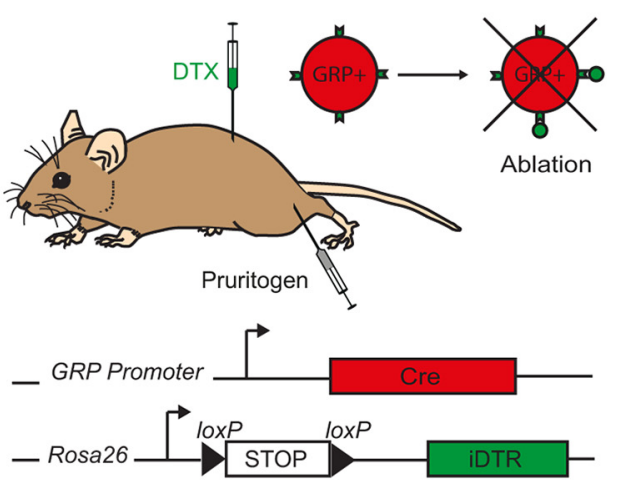

B

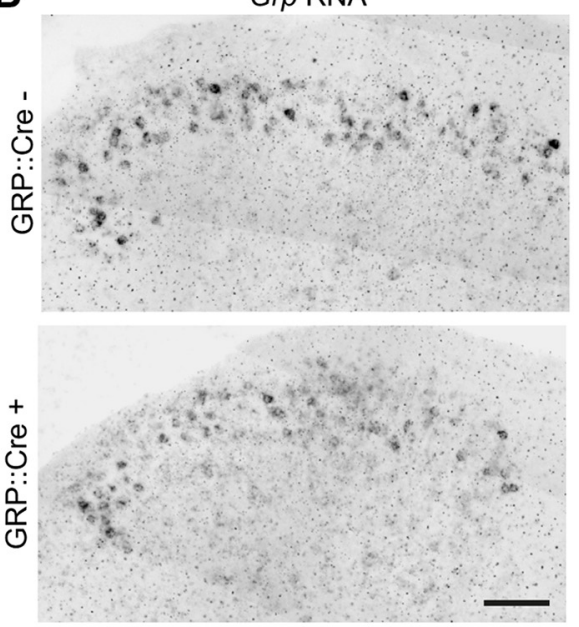

C

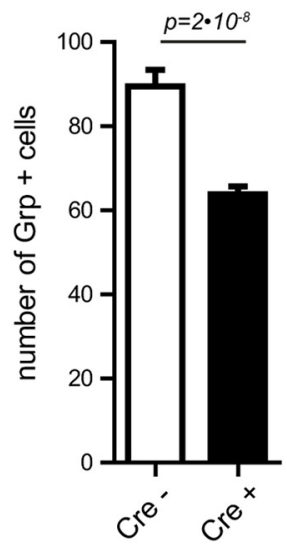

D

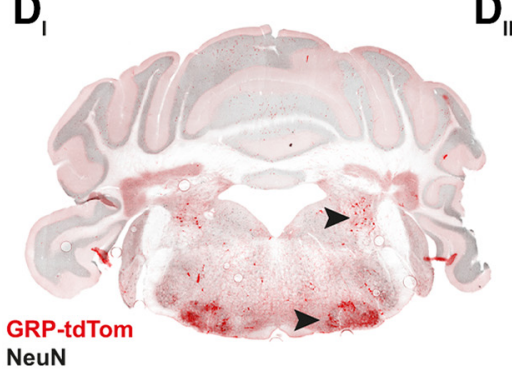

D II

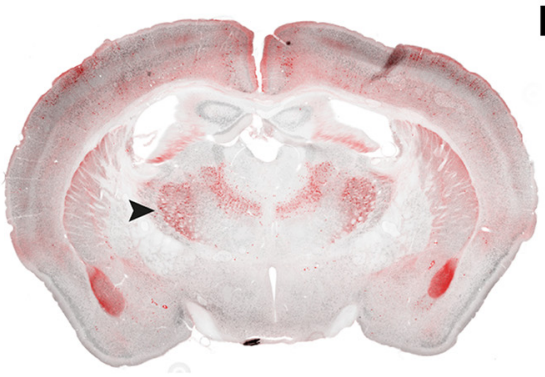

D

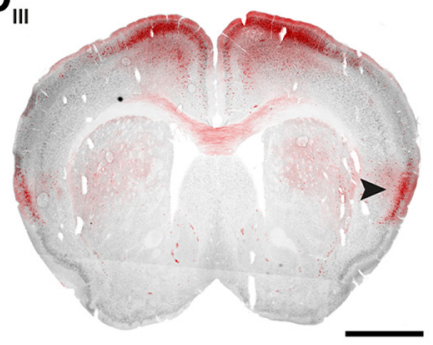

E

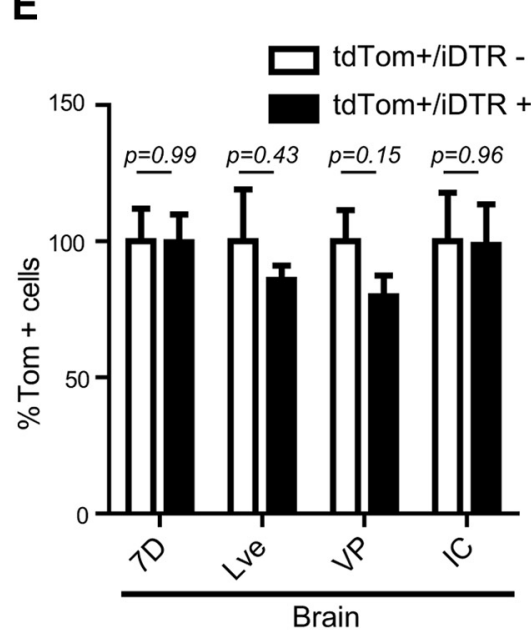

F

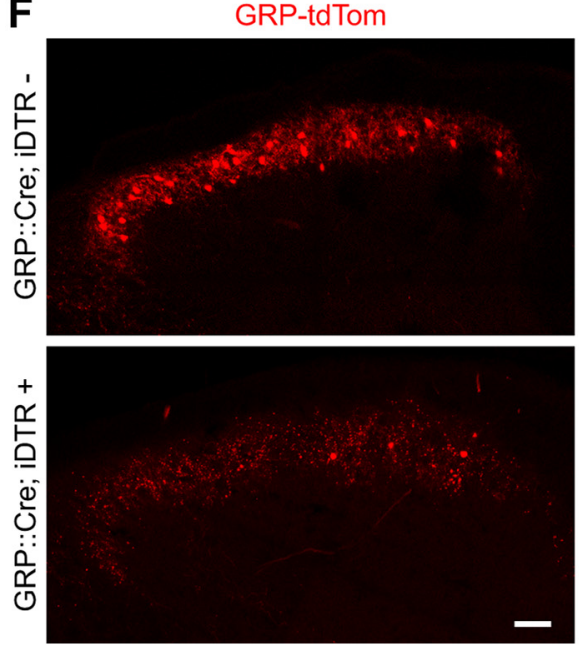

G

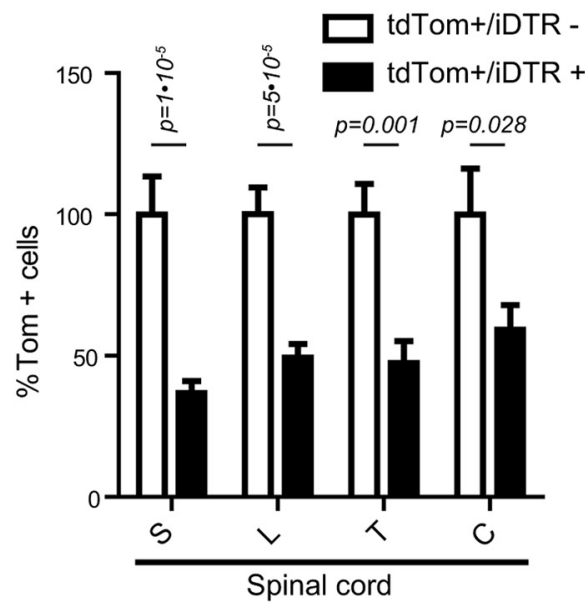

Figure 3. DTX-mediated ablation of GRP-cre neurons. $\boldsymbol{A}$, Strategy for targeted DTX-mediated ablation of GRP neurons. $\boldsymbol{B}$, ISH following intrathecal injection of DTX on spinal tissue of cre-negative control and GRP::Cre;ROSA26 $6^{\text {ISl-iDTR }}$ mice. Scale bar, $50 \mu \mathrm{m}$. $\boldsymbol{C}$, Quantification of $\boldsymbol{B}$, showing that Grp-expressing neurons are reduced by $29 \%$ following DTX-mediated ablation. $\boldsymbol{D}$, Coronal brain sections from GRP.: cre; ROSA26 $6^{\text {Isl-tdTom }}$ mouse showing abundant GRP-tdTom expression in several brain areas. Arrowheads indicate facial nucleus and lateral vestibular nucleus ( $\left.\boldsymbol{D}_{\boldsymbol{1}}\right)$, ventral posterior nucleus of the thalamus $\left(\boldsymbol{D}_{\text {II }}\right)$, and insular cortex $\left(\boldsymbol{D}_{\text {III) }}\right)$. Scale bar, $1 \mathrm{~mm}$. $\boldsymbol{E}$, Quantification of GRP-tdTom neurons at supraspinal sites following intrathecal injection of DTX in GRP:: ROSA26 $6^{\text {Isl-tdTom }}$ (tdTom ${ }^{+} /$iDTR $^{-}$) control mice and GRP::cre;ROSA26 $6^{\text {Isl-tdTom; }}$ ROSA26 $6^{\text {Isl-iDTR }}$ (tdTom ${ }^{+} /$iDTR $^{+}$) mice. Data are normalized by setting the number of cells counted in GRP::.cre; ROSA26 $6^{\text {Isl-tdTom }}$ control mice as $100 \%$. 7D, Facial nucleus; Lve, lateral vestibular nucleus; VP, ventral posterior nucleus of the thalamus; IC, insular cortex. $\boldsymbol{F}$, Confocal image of spinal dorsal horn

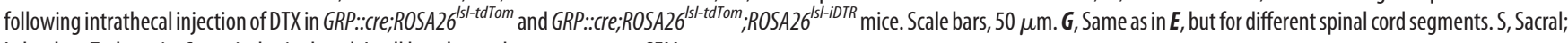
$\mathrm{L}$, lumbar, $\mathrm{T}$, thoracic; $\mathrm{C}$, cervical spinal cord. In all bar charts, data are mean $\pm \mathrm{SEM}$.

GRP-eGFP neurons to detect postsynaptic current responses upon optogenetic stimulation of MrgprA3 fibers (Fig. 2G); $4 \mathrm{~ms}$ blue light stimulation evoked EPSCs in 6 of 28 GRP-eGFP neurons (from 7 mice). Figure $2 \mathrm{H}$ shows individual EPSCs in a GRPeGFP neuron that responded to optogenetic stimulation of MrgprA3 fibers. Light-evoked EPSCs had on average amplitudes of $-126 \pm 57 \mathrm{pA}$ ( $n=6$ neurons from 5 mice), occurred with latencies of $12.8 \pm 0.18 \mathrm{~ms}$, jitters of $1.08 \pm 0.19 \mathrm{~ms}$ (Fig. $2 I, J$ ), and with low failure rates of $4.1 \pm 2.7 \%(n=6)$. Although the observed latencies were longer than latencies typically observed after electrical stimulation, their range is similar to that previously reported for light-evoked synaptic transmission between superficial dorsal horn neurons and unmyelinated MrgprD-positive fibers (Wang and Zylka, 2009). The low failure rate and the small jitter 
( $\leq 1.6 \mathrm{~ms}$ ) (Wang and Zylka, 2009) in 5 of 6 neurons provide further support for the monosynaptic nature of these signals.

We then went on with experiments to assess the behavioral consequences of manipulation of GRP neurons. We used two complementary strategies: DTX-mediated ablation and chemogenetic activation with the hM3Dq designer receptor exclusively activated by designer drugs (DREADDs). To this end, we first crossed the GRP:::cre mice with mice that express the DTX receptor (DTR) as a cre-dependent ("inducible") transgene (iDTR mice) to obtain triple transgenic GRP::cre;ROSA26 $6^{\text {lsl-tdTom; }}$ ROSA2 $6^{l s l-i D T R}$ mice. Because GRP neurons are also present at multiple supraspinal sites (http://www.gensat.org/cre.jsp), we chose to inject DTX ( $15 \mathrm{ng}$ in $3 \mu \mathrm{l}$ ) intrathecally in a hyperbaric ( $8 \%$ dextrose) solution to restrict ablation of GRP neurons to the spinal cord (Fig. 3A). Ten days after injection, we quantified the number of Grp mRNA-expressing neurons with ISH. We analyzed 18 and 72 sections from 3 Grp::cre-negative and 13 Grp::crepositive mice. Average numbers of Grp mRNA-positive neurons per section were $89.5 \pm 3.9$ and $63.8 \pm 1.8$, for Grp::cre-negative and 13 Grp::cre-positive mice, respectively $(t$ score $=5.94, p=$ $2 \times 10^{-8}$, unpaired $t$ test), corresponding to an average decrease of Grp mRNA-positive neurons by $29 \%$ (Fig. 3B,C). This percentage is consistent with the expression of GRP-cre in $\sim 25 \%$ of the Grp mRNA-positive neurons (compare also Fig. 1C). In line with previous reports about the supraspinal distribution of GRP neurons (Wada et al., 1990), we found GRP-tdTom-positive neurons (in GRP::cre;ROSA26 $6^{\text {lsl-tdTom }}$ mice) at various sites in the hindbrain and forebrain, including at least two areas relevant to central pain processing, that is, in the ventral posterior thalamic nuclei and the insular cortex (Fig. 3D; Table 2). We then compared the numbers of GRP-tdTom neurons after intrathecal DTX injection in brain sections of GRP::cre;ROSA26 $6^{\text {sl-tdTom; }}$ ROSA2 $^{\text {lsl-iDTR }}\left(\right.$ tdTom $\left.^{+} / \mathrm{iDTR}^{+}\right)$mice $(9$ sections per area from 3 mice) and of GRP:: cre;ROSA26 $6^{\text {lsl-tdTom }}\left(\right.$ tdTom $^{+} /$iDTR $\left.^{-}\right)$control mice (6 sections per area from 2 mice). Percentages of GRPtdTom neurons per section in DTX-injected iDTR-positive mice relative to DTX-injected but $i D T R$-negative control mice were $99.7 \pm 10.1 \%(t=0.02, p=0.99$, unpaired $t$ test $), 85.8 \pm 5.3 \%$ $(t=0.81, p=0.43), 79.9 \pm 7.6 \%(t=1.54, p=0.15)$, and $98.7 \pm$ $14.9 \%(t=0.06, p=0.96)$, for the facial nucleus, lateral vestibular nucleus, ventral posterior nucleus of the thalamus, and insular cortex, respectively (Fig. 3E). In the spinal cord, ablation occurred with a caudorostral gradient. The percentage of remaining GRP-tdTom neurons was lowest in the sacral (36.8 $\pm 4.3 \%$, $\left.t=5.93, p=1 \times 10^{-5}\right)$, intermediate in the lumbar $(49.3 \pm$ $4.8 \%, t=5.23, p=5 \times 10^{-5}$ ) (Fig. $3 F$ ) and thoracic segments (47.4 $\pm 7.8 \%, t=3.71, p=0.001)$, and highest in the cervical segment $(59.2 \pm 8.8 \%, t=2.38, p=0.028)$ (Fig. $3 G$ ).

We then continued with behavioral experiments to investigate the responses of spinal GRP neuron-ablated mice to several pruritoceptive stimuli (chloroquine, histamine, serotonin, and the PAR2 agonist SLIGRL) injected intracutaneously into the left calf (Fig. 4). Ten days after DTX injection, we observed a reduction in aversive behaviors (biting of the injected site) relative to GRP:: cre-negative ROSA26 $6^{\mid s l-i D T R}$ transgenic mice for three of the four pruritogens. The times spent biting were as follows: $154 \pm 18 \mathrm{~s}$ $(n=9)$ versus $104 \pm 11 \mathrm{~s}(n=9)$ for chloroquine-injected GRP::cre-negative and GRP::cre-positive mice $(t=2.30, p=0.03$, unpaired $t$ test); $382 \pm 40 \mathrm{~s}(n=8)$ and $272 \pm 30 \mathrm{~s}(n=9)$ for histamine-injected mice $(t=2.21, p=0.04)$; and $470 \pm 53 \mathrm{~s}(n=$ $10)$ and $313 \pm 37 \mathrm{~s}(n=10)$ for serotonin-injected mice $(t=2.45$, $p=0.02$ ) (Fig. $4 A-C$ ). The incomplete block of itch responses very likely reflects that $<30 \%$ of the Grp mRNA-positive neurons
Table 2. Distribution of GRP-tdTom-positive neurons in the mouse brain ${ }^{a}$

\begin{tabular}{ll}
\hline Brain area & Region \\
\hline Cortical plate & Lateral orbital cortex (LO) \\
& Ventral orbital cortex (V0) \\
& Agranular insular cortex (AI) \\
& Anterior cingulate cortex (Cg) \\
& Primary motor cortex (M1) \\
& Secondary motor cortex (M2) \\
& Somatosensory cortex (S1) \\
& Field CA3 hippocampus (CA3) \\
Cerebral nuclei & Accumbens nucleus (Acb) \\
Hypothalamus & Dorsomedial hypothalamic nucleus (DM) \\
& Suprachiasmatic nucleus (SCH) \\
Thalamus & Fields of Forel (FF) \\
& Anteroventral thalamic nucleus (AV) \\
& Anteromedial thalamic nucleus (AM) \\
& Ventral posterolateral thalamic nucleus (VPL) \\
& Ventral posteromedial thalamic nucleus (VPM) \\
& Mediodorsal thalamic nucleus (MDL) \\
& Centrolateral thalamic nucleus (CL) \\
& Medial geniculate nucleus (MG) \\
Anterior pretecal nucleus (APT) & Nucleus of the optic tract (OT) \\
Midbrain & Superior colliculus (SuG) \\
& Periaqueductal gray (PAG) \\
Oculomotor nucleus (3N) \\
Superior vestibular nucleus (SuVe) \\
Lateral vestibular nucleus (Lve) \\
Vacial nucleus (7N) \\
\hline
\end{tabular}

${ }^{a}$ Data are from 2 mice.

express cre. Unexpectedly, we did not observe a reduction in time spent biting for PAR2 (SLIGRL)-induced itch (290.7 $\pm 31.8 \mathrm{~s}$, $n=12$; vs $336 \pm 37 \mathrm{~s}, n=9$; for SLIGRL-injected mice; $t=0.94$, $p=0.36$, unpaired $t$ test (Fig. 4D). Given that several previous reports (Y.G. Sun and Chen, 2007; Y.G. Sun et al., 2009; Wan et al., 2017) found a reduction of SLIGRL-induced itch after interfering with GRP signaling pathways through different strategies, it is likely that, in our study, the GRP neurons remaining after DTX treatment account for the retained PAR2-induced pruritoceptive behavior.

Next, we used the same experimental approach to investigate potential effects of nociceptive (noxious heat, noxious cold, pinprick) and tactile (brush and von Frey) stimuli. Spinal GRP neuron-ablated mice and GRP::cre-negative control mice exhibited nearly identical response latencies and response scores (Hargreaves test latencies: $17.2 \pm 1.2 \mathrm{~s}, n=11$; vs $15.1 \pm 1.4 \mathrm{~s}, n=9$; for GRP:: cre-negative vs GRP::cre-positive mice; $t=1.14, p=$ 0.27 , unpaired $t$ test; cold plantar test latencies: $9.9 \pm 1.1 \mathrm{~s}, n=$ 11 ; vs $8.8 \pm 0.6 \mathrm{~s}, n=9 ; t=0.85, p=0.41$; pin prick response score: $87.5 \pm 6.2 \%, n=8$; vs $90.0 \pm 3.8 \%, n=7 ; t=0.33, p=$ 0.75; von Frey test threshold: $3.7 \pm 0.2 \mathrm{~g}, n=11$; vs $3.5 \pm 0.2 \mathrm{~g}$, $n=9 ; t=0.87, p=0.40$; brush test response score: $81.3 \pm 6.9 \%$, $n=8$; vs $95.7 \pm 3.0 \%, n=7 ; t=1.82, p=0.09$ ) (Fig. $4 E-I$ ). No impairment of motor coordination occurred in the rotarod test. Latencies to fall were $134 \pm 14 \mathrm{~s}(n=11)$ and $146 \pm 7 \mathrm{~s}(n=9)$, for control and GRP neuron ablated mice, respectively $(t=0.76$, $p=0.46$, unpaired $t$ test, data not shown).

In the second, complementary approach, we locally activated spinal GRP neurons using an excitatory chemogenetic strategy. The left lumbar dorsal horn of GRP::cre transgenic mice was injected with an adeno-associated virus (AAV) carrying a cassette for cre-dependent expression of the excitatory DREADD hM3Dq (Alexander et al., 2009) fused in frame with red fluorescent pro- 
A
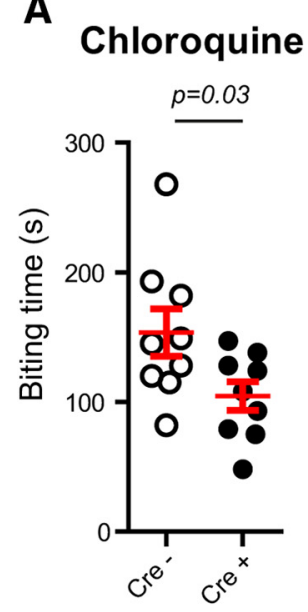

E

Hargreaves

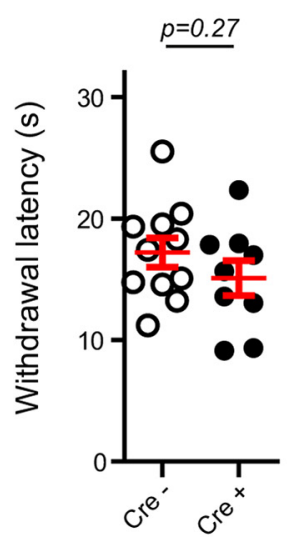

B

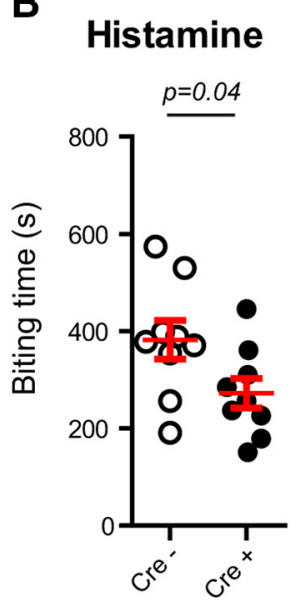

$\mathbf{F}$

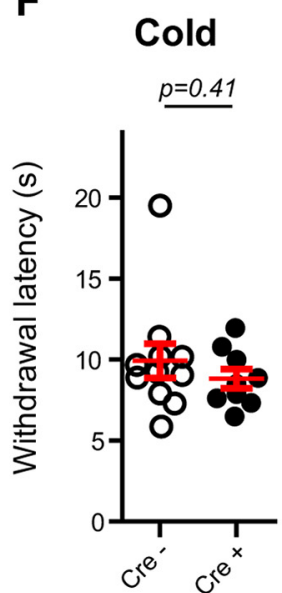

C

Serotonin

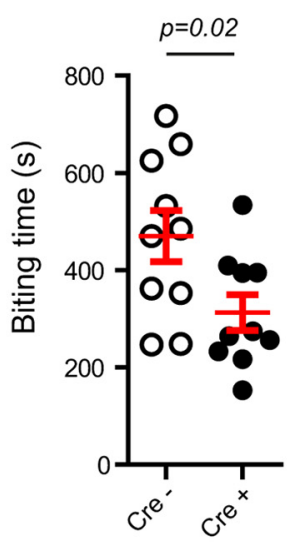

G

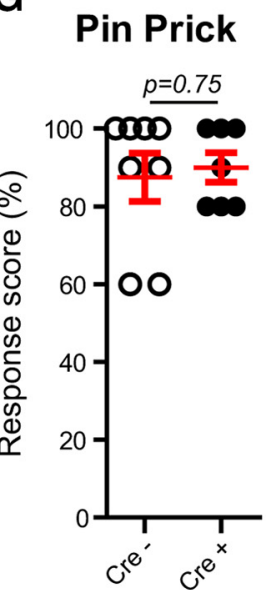

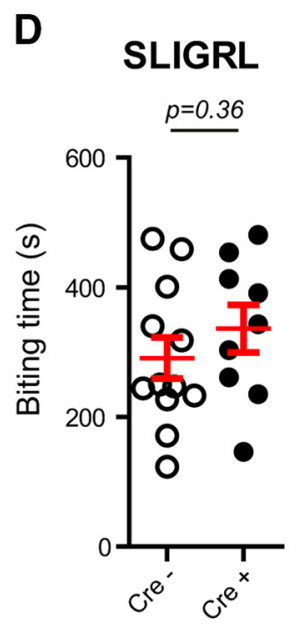

H
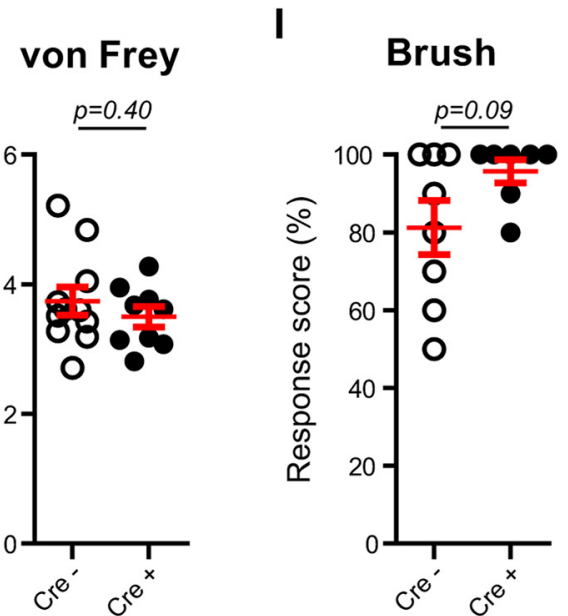

Figure 4. Behavioral effects of DTX-mediated ablation of GRP-cre neurons. A-D, Reduced itch responses to chloroquine, histamine, and serotonin but not SLIGRL following DTX-mediated ablation of GRP neurons. $E-I$, Unaltered thermal and mechanical nociceptive responses in GRP neurons ablated mice. Circles represent measurements from individual mice. Error bars indicate mean \pm SEM.

tein mCherry (Fig. 5A). hM3Dq permitted selective activation of GRP neurons by CNO. Seven days after virus injection, mCherry expression was detected in lamina II neurons of the injected dorsal horn (Fig. 5B). Before CNO injection, expression of hM3Dq did not induce any scratching or biting behavior beyond what was observed in cre-negative AAV1.hSyn.flex.hM3Dq-mCherryinjected mice (Fig. $5 C$ ). However, the same mice responded with intense aversive behaviors (biting of the skin territories innervated by the injected spinal segment) when injected systemically (intraperitoneally) with $\mathrm{CNO}(2 \mathrm{mg} / \mathrm{kg}$ ) (Fig. $5 C, D)$. No such effects were observed in cre-negative mice that had undergone the same virus injection, strongly suggesting that activity of spinal GRP neurons evokes profound aversive behaviors reminiscent of responses elicited by acute pruritic stimuli. Time spent biting was $16.6 \pm 8.5 \mathrm{~s}(n=9)$ and $195 \pm 35 \mathrm{~s}(n=6)$, for GRP::cre-negative and GRP::cre-positive mice $\left(t=6.03, p=4 \times 10^{-5}\right.$, unpaired $t$ test) (Fig. 5C,D).

Our results described above are consistent with a specific role of spinal GRP neurons in itch. This specificity has recently been challenged when S. Sun et al. (2017) proposed that spinal GRP neurons would also contribute to nociceptive signal relay. Unlike itch relay, this nociceptive relay would follow a bell-shaped response curve with significant contributions occurring only at low activity levels. More intense activity of spinal GRP neurons would close the spinal pain gate. To rule out the possibility that we had missed an effect on pain behaviors because our chemogenetic stimulation was too strong, we investigated possible potentiating actions of $\mathrm{CNO}$ on responses evoked by nociceptive or pruritoceptive stimulation at a later time point when "spontaneous" CNO-induced pruritoceptive responses had subsided (Fig. $5 C, E-K$, red and blue traces). One group of mice was challenged with either chloroquine $(80 \mu \mathrm{g}$ in $10 \mu \mathrm{l})$ or histamine $(100 \mu \mathrm{g}$ in $10 \mu \mathrm{l})$ to assess a potential facilitation of itch responses. Responses to both pruritogens were significantly higher in the crepositive AAV1.hSyn.flex.hM3Dq-mCherry injected mice than in cre-negative mice, indicating that at this time point the remaining $\mathrm{CNO}$ concentration was high enough to increase the excitability of the GRP neurons (times spent biting for chloroquine: $135 \pm 21 \mathrm{~s}, n=14$; vs $269 \pm 31 \mathrm{~s}, n=11 ; t=3.78, p=0.001$, unpaired $t$ test; for GRP::cre-negative and GRP::cre-positive mice, respectively; for histamine: $189 \pm 30 \mathrm{~s}, n=12$; vs $361 \pm 58 \mathrm{~s}, n=$ $11 ; t=2.73, p=0.01$ ) (Fig. $5 C, E, F)$. By contrast, we failed to detect a change in the responses to noxious stimuli (Hargreaves test latencies: $24.1 \pm 2.0 \mathrm{~s}, n=12$; vs $20.1 \pm 1.3 \mathrm{~s}, n=12$; $t=1.38$, $p=0.18$, unpaired $t$ test, for GRP::cre-negative and GRP::crepositive mice, respectively; noxious cold test latencies: $12.7 \pm$ $1.2 \mathrm{~s}, n=12$; vs $13.8 \pm 1.2 \mathrm{~s}, n=11 ; t=0.69, p=0.49$; pin-prick response score: $89.2 \pm 5.7 \%, n=12$; vs $92.5 \pm 3.3 \%, n=12$; $t=$ 
A

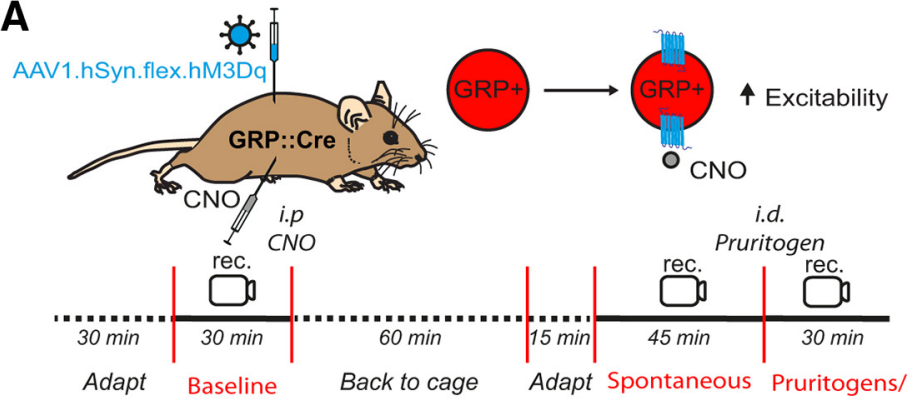

C

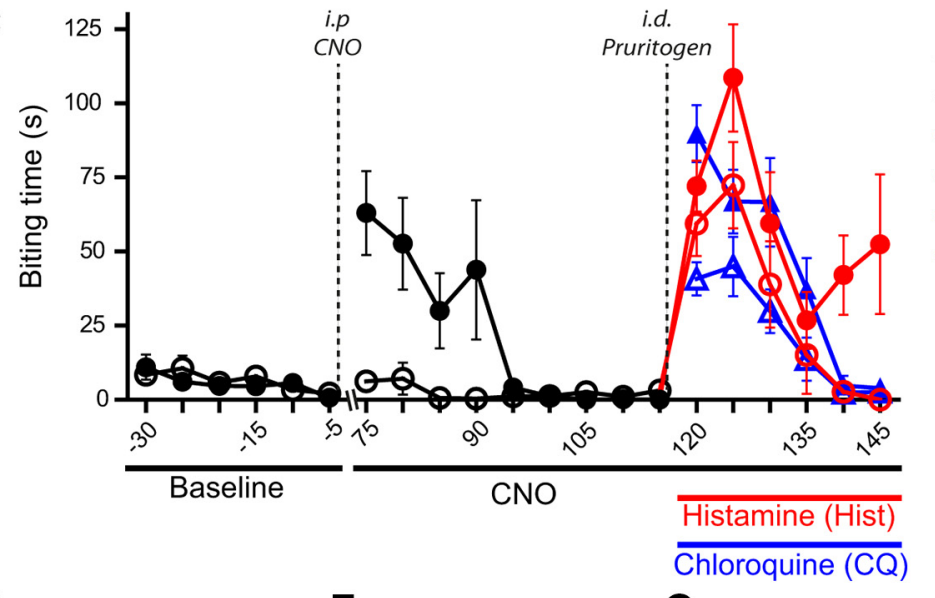

E Chloroquine
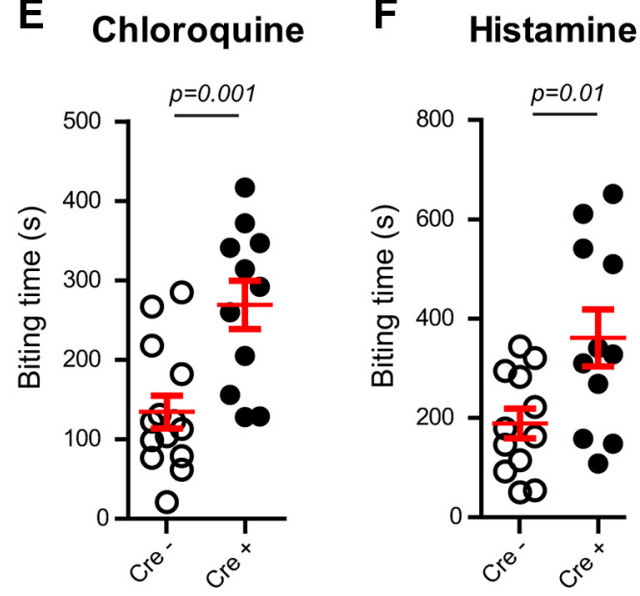

J
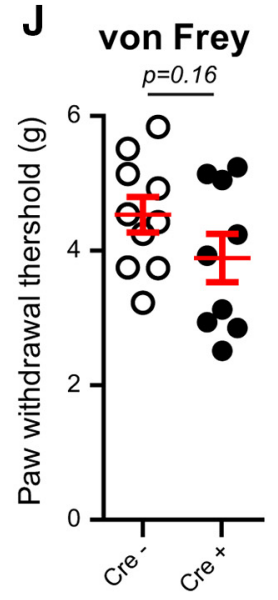

K

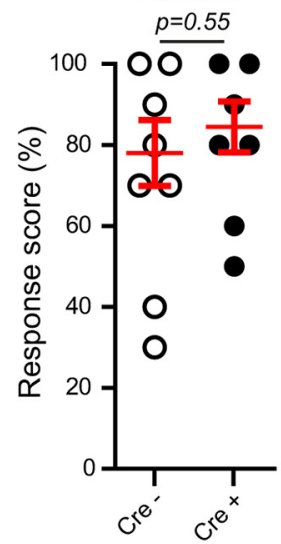

B

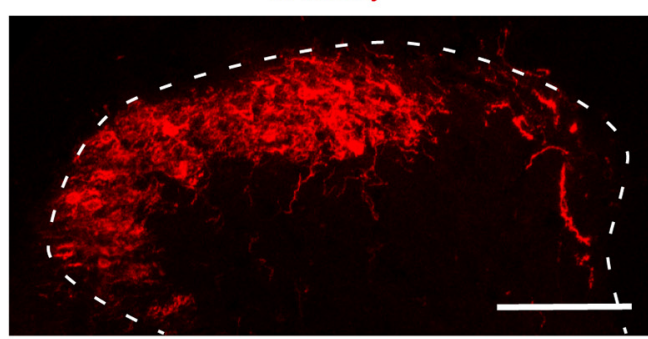

O Cre -

- Cre+

Cre-, Hist

$\multimap$ Cre +, Hist

$\triangle$ Cre-, CQ

$\longrightarrow \mathrm{Cre}+\mathrm{CQ}$

D Spontaneous
G Hargreaves

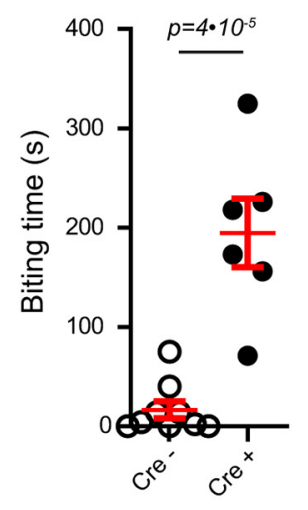

H

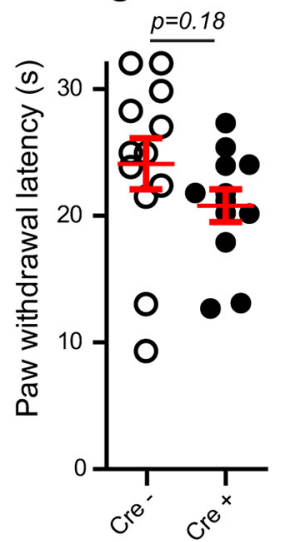

L

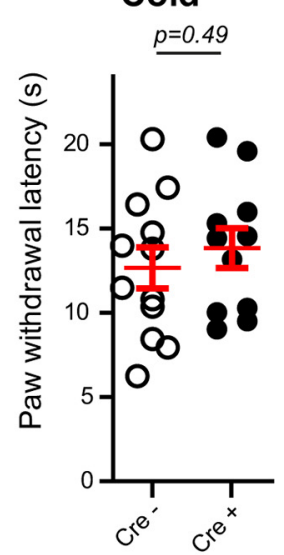

M
I Pin Prick

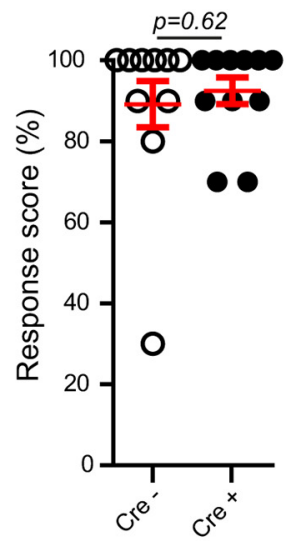

Figure 5. Chemogenetic activation of GRP-cre neurons. $\boldsymbol{A}$, Strategy for DREADD-mediated activation of GRP-cre neurons and timeline of the experiment design. $\boldsymbol{B}$, Spinal section showing cre-dependent hM3D-mCherry expression in GRP-cre neurons. Scale bar, $100 \mu \mathrm{m}$. C, Time course of itch behavior before and after DREADD-mediated activation of GRP-cre neurons. CNO induces itch response in GRP::cre mice intraspinally injected with AAV1.hSyn.flex.hM3Dq-mCherry but not in control cre-negative mice. Pruritogens histamine and chloroquine were injected intradermally 120 min after CNO treatment. Chemogenetic activation of GRP neurons increases pruritogen-induced biting behavior. Biting behavior was quantified in intervals of 5 min. (Figure legend continues.) 
HSV-Tomato NeuN
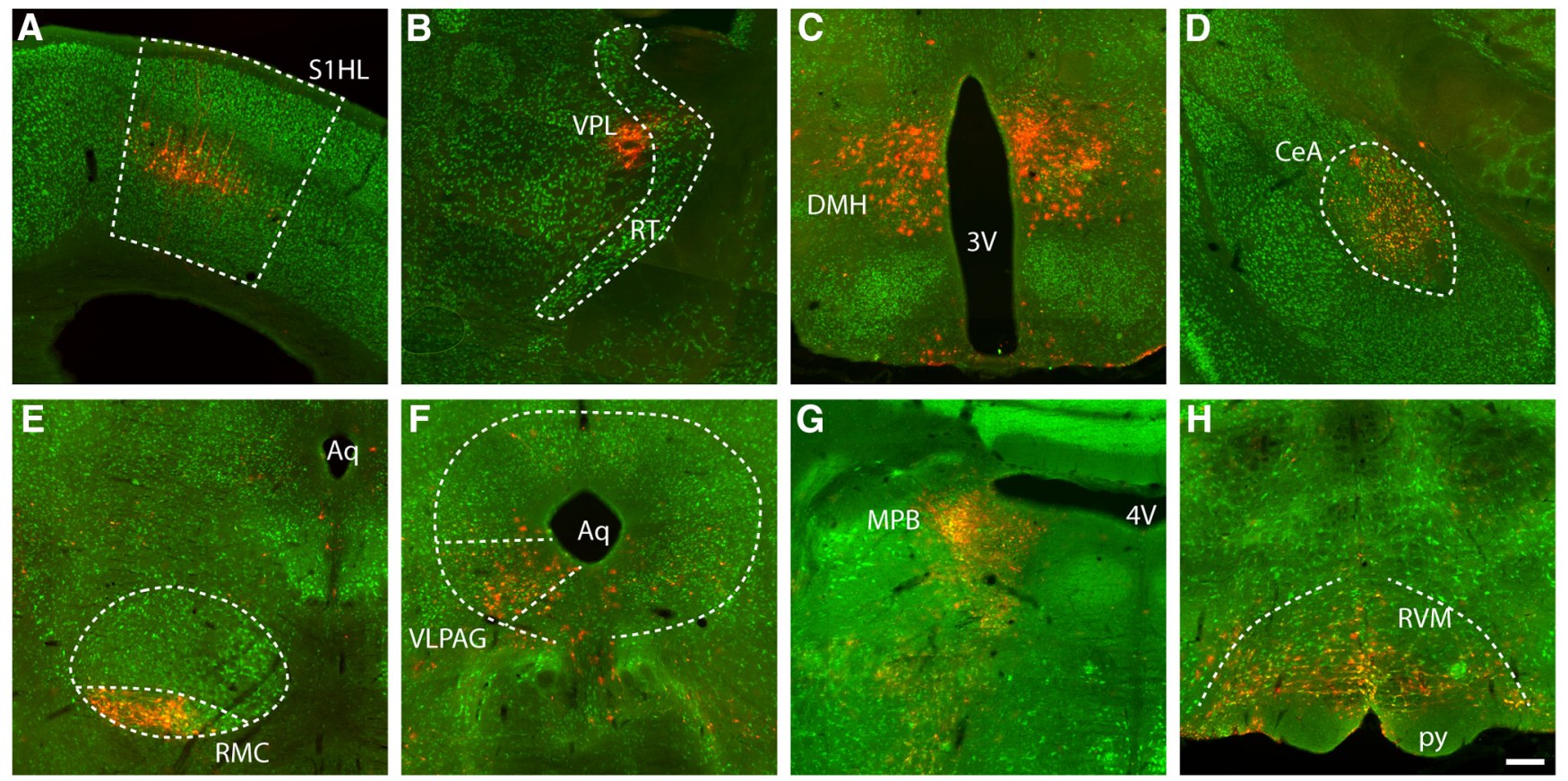

Figure 6. HSV-based anterograde tracing initiated from spinal GRP-cre neurons. Immunostaining on coronal brain sections of GRP::cre mice intraspinally injected with HSV showing brain areas receiving polysynaptic connections from GRP neurons, including the following: $(\boldsymbol{A})$ somatosensory cortex hindlimb region $(\mathrm{S} 1 \mathrm{HL}),(\boldsymbol{B})$ ventral posterolateral nucleus of the thalamus $(\mathrm{VPL}),(\boldsymbol{C})$ dorsomedial hypothalamic nucleus (DMH), (D) central nucleus of the amygdala (CeA), $(\boldsymbol{E})$ red nucleus magnocellular part (RMC), $(\boldsymbol{F})$ ventrolateral periaqueductal gray (VLPAG), $(\boldsymbol{G})$ medial parabrachial nucleus (MPB), and (H) rostral ventromedial medulla (RVM). Scale bar, $200 \mu \mathrm{m}$.

$0.51, p=0.62$ ) (Fig. $5 G-I$ ), confirming the lack of an effect of GRP neuron activation on pain. Similarly, no changes were observed for innocuous tactile stimulation (von Frey thresholds: $4.5 \pm 0.3 \mathrm{~g}, n=10$; vs $3.9 \pm 0.4 \mathrm{~g}, n=9 ; t=1.47, p=0.16$; brush response score: $78.0 \pm 8.1 \%, n=10$; vs $84.4 \pm 6.3 \%, n=9$; $t=$ $0.62, p=0.55$ ) (Fig. $6 \mathrm{~J}, K$ ). No impairment of motor coordination was observed in the rotarod test. Latencies to fall were $103 \pm$ $13 \mathrm{~s}$ and $127 \pm 11 \mathrm{~s}$, for $\mathrm{CNO}$-injected cre-negative (control) and cre-positive mice, respectively $(t=1.34, p=0.2$, unpaired $t$ test, data not shown).

In a second approach, we used different doses of $\mathrm{CNO}(0.7$, 0.1 , and $0.02 \mathrm{mg} / \mathrm{kg}$, i.p.) and compared their effects on spontaneous itch-like behavior. We found a dose-dependent increase in the times spent biting $(0.7 \mathrm{mg} / \mathrm{kg}: 35.7 \pm 13.1 \mathrm{~s}, n=6$ GRP::crenegative; vs $227 \pm 49 \mathrm{~s}, n=6$ GRP::cre-positive mice; $t=3.79$, $p=0.004$, unpaired $t$ test; $0.1 \mathrm{mg} / \mathrm{kg}: 31.3 \pm 7.1 \mathrm{~s}, n=6$; vs $139 \pm$ $41 \mathrm{~s}, n=6 ; t=2.61, p=0.03 ; 0.02 \mathrm{mg} / \mathrm{kg}: 17.7 \pm 7.2 \mathrm{~s}, n=6$; vs $41.5 \pm 18.2 \mathrm{~s}, n=6 ; t=1.22, p=0.25$ ) (Fig. $5 L$ ), but no effects on response latencies in the Hargreaves test $(0.7 \mathrm{mg} / \mathrm{kg}: 16.9 \pm$ 3.0 s, GRP::cre-negative; vs $16.8 \pm 2.3$ s, GRP::cre-positive mice; $t=0.02, p=0.99$, unpaired $t$ test; $0.1 \mathrm{mg} / \mathrm{kg}: 12.1 \pm 1.4 \mathrm{~s}$ vs

$\longleftarrow$

(Figure legend continued.) Timeline on the $x$ axis indicates the time from CNO intraperitoneal injection. $\boldsymbol{D}$, Chemogenetic activation of spinal GRP-cre neurons induced itch response. Biting behavior was quantified for $30 \mathrm{~min}$ starting $75 \mathrm{~min}$ after CNO injection. $\boldsymbol{E}, \boldsymbol{F}$, Increased histamine- and chloroquine-induced itch behavior following chemogenetic activation of spinal GRP-cre neurons. Biting behavior was quantified from 120 min after CNO treatment, for $30 \mathrm{~min}$. $\boldsymbol{G}-\boldsymbol{K}$, Unaltered nociceptive responses following chemogenetic activation of spinal GRP-cre neurons. $\boldsymbol{L}, \boldsymbol{M}$, Dose-dependent induction of spontaneous pruritoceptive (biting) responses by $\mathrm{CNO}$, but no sensitization to heat in the Hargreaves test. Measurements were taken 75 min after CNO injection. Circles represent measurements from individual mice. Error bars indicate mean \pm SEM.
$13.3 \pm 1.6 \mathrm{~s}, t=0.52, p=0.61 ; 0.02 \mathrm{mg} / \mathrm{kg}: 19.2 \pm 2.8 \mathrm{~s}$ vs $19.3 \pm$ $0.9 \mathrm{~s}, t=0.01, p=0.99, n=6$ for all groups) (Fig. $5 M$ ). Together, these results suggest that spinal GRP neurons do not exert an apparent function in spinal pain control.

Finally, we used polysynaptic anterograde virus-based tracing to investigate supraspinal CNS areas innervated by neuronal pathways emerging from GRP neurons (Fig. 6). To this end, we injected the cre-dependent H129DTK-TT herpes virus variant (Lo and Anderson, 2011) into the lumbar spinal cord of 3 GRP:: cre mice. H129DTK-TT becomes recombined in cre-expressing neurons to enable replication and tdTomato expression first in the cre-expressing neurons and subsequently in all neurons connected in an anterograde manner. Four to five days after HSV injection, mice were perfused and coronal sections were taken at $240 \mu \mathrm{m}$ intervals. tdTom expression occurred at several distinct supraspinal sites (Table 3). Among the tdTom-labeled sites were the medial parabrachial nucleus, the rostral ventromedial medulla, the ventrolateral periaqueducal gray, the red nucleus, the dorsomedial hypothalamus, the sensory thalamic nuclei, the central amygdala, and the somatosensory cortex (hindlimb region). Several of these areas have previously been identified in functional brain imaging experiments on itch (for review, see Forster and Handwerker, 2014). Our findings thus confirm the results of the imaging studies and further support the role of GRP neurons in itch.

\section{Discussion}

The presence of peripheral sensory neurons whose activation specifically elicits itch-like behavior (Liu et al., 2009; Mishra and Hoon, 2013; Huang et al., 2018) and the enriched expression in these neurons of receptors activated by pruritogens (Usoskin et al., 2015; Nguyen et al., 2017) supports the concept of a "labeled line" for itch at the level of the primary sensory neurons. Such a 
Table 3. Supraspinal CNS regions anterogradely traced from lumbar spinal cord GRP neurons ${ }^{a}$

\begin{tabular}{|c|c|c|}
\hline Brain area & Region & Stained cells \\
\hline \multirow[t]{2}{*}{ Cortical plate } & Agranular insular cortex (Al) & + \\
\hline & Primary somatosensory cortex hindlimb region (S1HL) & ++ \\
\hline Cortical subplate & Central nucleus of the amygdala (CeA) & + \\
\hline Pallidum & Extended amygdala (EA) & + \\
\hline \multirow[t]{4}{*}{ Hypothalamus } & Medial preoptic nucleus (MP0) & + \\
\hline & Paraventricular hypothalamic nucleus posterior part ( $\mathrm{PaP} 0)$ & +++ \\
\hline & Dorsomedial hypothalamic nucleus (DM) & ++ \\
\hline & Lateral hypothalamic area (LHA) & + \\
\hline \multirow[t]{8}{*}{ Thalamus } & Paraventricular thalamic nucleus anterior part (PVA) & + \\
\hline & Paratenial thalamic nucleus (PT) & + \\
\hline & Ventrolateral thalamic nucleus (VL) & ++ \\
\hline & Paraventricular thalamic nucleus (PV) & + \\
\hline & Mediodorsal thalamic nucleus medial part (MDM) & + \\
\hline & Interanteromedial thalamic nucleus (IAM) & + \\
\hline & Midline thalamic nuclei (MTN) & + \\
\hline & Ventromedial thalamic nucleus (VM) & + \\
\hline \multirow[t]{8}{*}{ Midbrain } & Red nucleus (R) & +++ \\
\hline & Periaqueductal gray (PAG) & ++ \\
\hline & Ventrolateral periaqueductal gray (VLPAG) & ++ \\
\hline & Mesencephalic reticular formation (mRt) & ++ \\
\hline & Median raphe nucleus (MRN) & + \\
\hline & Isthmic reticular formation (isRT) & ++ \\
\hline & Superior colliculus (SC) & + \\
\hline & Dorsal raphe (DR) & +++ \\
\hline \multirow[t]{4}{*}{ Pons } & Pontine nuclei (Pn) & + \\
\hline & Locus coeruleus (LC) & +++ \\
\hline & Medial parabrachial nucleus (MPB) & +++ \\
\hline & Lateral parabrachial nucleus (LPB) & ++ \\
\hline \multirow[t]{2}{*}{ Cerebellar nuclei } & Deep cerebellar nuclei (DCN) & ++ \\
\hline & Lateral cerebellar nucleus (Lat) & + \\
\hline \multirow[t]{5}{*}{ Medulla } & Intermediate reticular nucleus (iRt) & ++ \\
\hline & Raphe magnus nucleus (RMg) & ++ \\
\hline & Spinal vestibular nucleus (SpVe) & ++ \\
\hline & Raphe obscurus nucleus (Rob) & +++ \\
\hline & Lateral paragigantocellular nucleus (LPGi) & ++ \\
\hline
\end{tabular}

${ }^{a}$ Data are from 3 mice. + , low density; ++ , high density; +++ , very high density.

peripheral labeled line is in agreement with microneurography experiments that revealed a specific class of $\mathrm{C}$ fibers that become tonically active upon exposure to histamine (Schmelz et al., 1997). Less clear is whether this labeled line also exists at the spinal cord level. The well-established interaction of nociceptive and pruritoceptive pathways at the level of spinal cord (Liu et al., 2010; Kardon et al., 2014; S. Sun et al., 2017) has given rise to the selectivity, as opposed to specificity, theory of itch (Ma, 2010). Here, we have focused our efforts on the GRP-positive population of dorsal horn neurons. Our study identifies these neurons as a rather homogeneous population of lamina II excitatory interneurons with a central cell-like morphology. These results are generally in good agreement with several previous studies (Gutierrez-Mecinas et al., 2014, 2016a; Solorzano et al., 2015; S. Sun et al., 2017) that found that GRP expression defines a distinct set of excitatory dorsal horn neurons. Our multicolor fluorescence confocal microscopy results and the optogenetic experiments in spinal cord slices revealed that GRP neurons receive excitatory synaptic input from the MrgprA3-positive population of primary pruritoceptors. This innervation pattern correlates well with our behavioral experiments in which we found that manipulation of GRP neurons interfered with behavioral responses to pruritic stimuli but did not change response to acute painful stimuli. They hence support previous studies that attributed to GRP receptors and GRPR-expressing neurons specific roles in itch (Y.G. Sun and Chen, 2007; Y.G. Sun et al., 2009). As such, our results support that the concept of a "labeled line" for itch might extend from the periphery at least to second-order spinal neurons. This is also supported by a recent study that reported little capsaicin-sensitive (nociceptive) synaptic input onto GRP neurons (Dickie et al., 2019). Nevertheless, all of the above findings are consistent with selectivity theory of itch and the well-established cross talk from nociceptive to pruritoceptive relay pathways (Kardon et al., 2014).

Recently, the "leaky gate" model has been proposed as an additional alternative to the labeled line or selectivity concepts (S. Sun et al., 2017). This model proposes that GRP neurons would receive input from sensory fibers that elicit itch sensations and from other fibers that trigger pain responses. The GRP neurons would then convey itch signals and low-intensity pain signals, whereas high-intensity pain signals would be blocked through a GRP neuron-evoked feedforward inhibition. This intensity-dependent feedforward inhibition would lead to a normal stimulus response curve for GRP neuron-mediated itch but to a bell-shaped response curve for pain. In our experiments, we did not find evidence for this scenario. One might argue that the intensity of chemogenetic activation of GRP neurons in our experiments was too strong to evoke pain responses. However, subthreshold activation of GRP neurons by CNO still only facilitated responses to pruritoceptive, but not to nociceptive, stimuli (compare Fig. $5 L, M)$. Furthermore, our ablation experiments also failed to provide evidence for an involvement of GRP neurons in pain. Because both the study by S. Sun et al. (2017) and our study used the same line of GRP::cre BAC transgenic mice and the same iDTR mouse line for ablation, differences in transgene expression can largely be ruled out as an explanation of the different results. One potentially relevant difference in the experimental design, however, is the route of administration of DTX. In our experiments, we injected DTX locally into the intrathecal space of the lumbar spinal cord and in a hyperbaric solution to prevent diffusion to the brain. S. Sun et al. (2017), by contrast, applied DTX systemically via (intraperitoneal) injections. This difference may be relevant, as the presence of GRP neurons is not restricted to the spinal cord. Indeed, the GRP::cre mouse line used in both studies shows strong expression in several brain regions, including the somatosensory cortex and the thalamus (http://www.gensat.org/ creGeneView.jsp?founder_id=64533\&gene_id=597) consistent with our findings obtained with the Ai14 reporter mice. It is very likely that the systemic administration of DTX-targeted GRP neurons also at supraspinal sites, whereas the intrathecal injection of hyperbaric DTX solutions confined ablation to the spinal cord (compare Fig. 3). Ablation of GRP neurons at several sites might impact on the pain sensitivity of mice. First, the anterior cingulate cortex and the insular cortex serve pivotal functions in pain sensation and hyperalgesia. Second, the ventral posterior thalamic nuclei are well-established relay stations of nociceptive signals; and, finally, GRP neurons in the suprachiasmatic nucleus of the hypothalamus serve pivotal functions in circadian biology and sleep. Administration of GRP at this site causes phase shifts in circadian behavior (Piggins et al., 1995). Ablation of these neurons may impair physiological sleep-wake cycles, which have recently been shown to induce states of hyperalgesia (Alexandre et al., 2017). Although the precise role in pain of the GRP neurons at these sites remains to be defined, it cannot be excluded that their ablation impairs pain responses.

A second potentially relevant difference between the two studies is the extent of GRP neuron activation. We have used local AAV-mediated gene transfer to express hM3Dq in GRP neurons 
locally in three unilateral segments of the lumbar spinal cord. Activation was hence confined to GRP neurons of the dorsal horn of only three lumbar segments of one side of the spinal cord (for an illustration of the extent of AVV-mediated gene transfer, see Foster et al., 2015). S. Sun et al. (2017) expressed the capsaicin receptor TRPV1 globally in all GRP-positive cells and activated GRP neurons via intrathecal capsaicin injection in a nonhyperbaric solution. This procedure excites GRP neurons bilaterally and likely over a large part of the spinal cord potentially extending even to the brainstem or brain. Finally, TRPV1 and hM3Dq activate neurons via very different cellular signaling pathways (i.e., either via direct gating of an ion channel or via activation of a GPCR) and with different kinetics. It is conceivable that they result in different firing patterns that may impact on downstream release of fast or peptide neurotransmitters and on the decoding of the postsynaptic signals, such as itch or pain.

Up to date, relatively little information is available about supraspinal CNS areas involved in the processing of pruritoceptive signals. While brain imaging (fMRI) and PET studies in human subjects have been instrumental in defining brain areas processing sensory information in the visual, auditory, and nociceptive system, their application in itch research is complicated by the slow onset, and the waning and waxing nature of itch sensations (Forster and Handwerker, 2014). Brain areas reported to be activated during exposure to different pruritic stimuli (or silenced upon itch suppression) include prefrontal areas, insular cortex, cingulate cortex, somatosensory cortex, thalamus, basal ganglia, and cerebellum (Leknes et al., 2007; Papoiu et al., 2014, 2015; see also Forster and Handwerker, 2014). Many of these areas were also labeled in our virus-based anterograde tracing experiment that started from GRP neurons. These include the insular cortex, sensory parts of the thalamus, the somatosensory cortex, the cerebellum, the red nucleus, and the amygdala. Some differences between the results of our tracing studies and previous imaging experiments may be due to the specific incubation time of $4-5 \mathrm{~d}$ after virus injection, which might have been too short to trace the most rostral elements of the itch pathway, such as the anterior cingulate cortex. On the other hand, our tracing study has identified specific labeling in certain deep brain structures and structures in the hindbrain that are difficult to detect with fMRI. These include the paraventricular and dorsomedial hypothalamic nuclei, the ventrolateral periaqueductal gray, the locus ceruleus, the raphe nuclei, and the parabrachial nucleus that has recently been described as the first supraspinal relay site for itch signals (Mu et al., 2017). Given the aversive nature of both itch and pain, it is not surprising that brain regions activated in response to painful or pruritic stimulation show big overlap (Forster and Handwerker, 2014; Vierow et al., 2015). In case of the herpes simplex-mediated anterograde tracing, however, it needs to be considered that HSV is propagated not only along excitatory pathways but also via inhibitory connections, potentially leading to the labeling of pathways that are suppressed by itch.

In conclusion, our results demonstrate that dorsal horn GRP neurons constitute a rather homogeneous population of excitatory interneurons serving a critical role in the spinal relay of itch signals. Our chemogenetic silencing experiments and diphtheria toxin-mediated ablation of GRP neurons do not support a role of these neurons in pain. The anatomical tracing experiments reveal that the polysynaptic long-range projections that originate from GRP neurons overlap with areas identified in functional brain imaging experiments.

\section{References}

Albisetti GW, Ghanem A, Foster E, Conzelmann KK, Zeilhofer HU, Wildner H (2017) Identification of two classes of somatosensory neurons that display resistance to retrograde infection by rabies virus. J Neurosci 37:10358-10371.

Alexander GM, Rogan SC, Abbas AI, Armbruster BN, Pei Y, Allen JA, Nonneman RJ, Hartmann J, Moy SS, Nicolelis MA, McNamara JO, Roth BL (2009) Remote control of neuronal activity in transgenic mice expressing evolved G-protein-coupled receptors. Neuron 63:27-39.

Alexandre C, Latremoliere A, Ferreira A, Miracca G, Yamamoto M, Scammell TE, Woolf CJ (2017) Decreased alertness due to sleep loss increases pain sensitivity in mice. Nat Med 23:768-774.

Brenner DS, Golden JP, Gereau RW 4th (2012) A novel behavioral assay for measuring cold sensation in mice. PLoS One 7:e39765.

Chung K, Deisseroth K (2013) CLARITY for mapping the nervous system. Nat Methods 10:508-513.

Dickie AC, Bell AM, Iwagaki N, Polgar E, Gutierrez-Mecinas M, Kelly R, Lyon H, Turnbull K, West SJ, Etlin A, Braz J, Watanabe M, Bennett DL, Basbaum AI, Riddell JS, Todd AJ (2019) Morphological and functional properties distinguish the substance $\mathrm{P}$ and gastrin-releasing peptide subsets of excitatory interneuron in the spinal cord dorsal horn. Pain 160:442-462.

Doyle MW, Andresen MC (2001) Reliability of monosynaptic sensory transmission in brain stem neurons in vitro. J Neurophysiol 85:22132223.

Dugué GP, Brunel N, Hakim V, Schwartz E, Chat M, Lévesque M, Courtemanche R, Léna C, Dieudonné S (2009) Electrical coupling mediates tunable low-frequency oscillations and resonance in the cerebellar Golgi cell network. Neuron 61:126-139.

Forster C, Handwerker HO (2014) Central nervous processing of itch and pain. In: Itch: mechanisms and treatment (Carstens E, Akiyama T, eds). Boca Raton, FL: CRC.

Foster E, Wildner H, Tudeau L, Haueter S, Ralvenius WT, Jegen M, Johannssen H, Hösli L, Haenraets K, Ghanem A, Conzelmann KK, Bösl M, Zeilhofer HU (2015) Targeted ablation, silencing, and activation establish glycinergic dorsal horn neurons as key components of a spinal gate for pain and itch. Neuron 85:1289-1304.

Gutierrez-Mecinas M, Watanabe M, Todd AJ (2014) Expression of gastrinreleasing peptide by excitatory interneurons in the mouse superficial dorsal horn. Mol Pain 10:79.

Gutierrez-Mecinas M, Furuta T, Watanabe M, Todd AJ (2016a) A quantitative study of neurochemically defined excitatory interneuron populations in laminae I-III of the mouse spinal cord. Mol Pain 12: 1744806916629065.

Gutierrez-Mecinas M, Kuehn ED, Abraira VE, Polgár E, Watanabe M, Todd AJ (2016b) Immunostaining for homer reveals the majority of excitatory synapses in laminae I-III of the mouse spinal dorsal horn. Neuroscience 329:171-181.

Haenraets K, Albisetti GW, Foster E, Wildner H (2018) Adeno-associated virus-mediated transgene expression in genetically defined neurons of the spinal cord. J Vis Exp 12:135.

Han L, Ma C, Liu Q, Weng HJ, Cui Y, Tang Z, Kim Y, Nie H, Qu L, Patel KN, Li Z, McNeil B, He S, Guan Y, Xiao B, Lamotte RH, Dong X (2013) A subpopulation of nociceptors specifically linked to itch. Nat Neurosci 16:174-182.

Huang J, Polgár E, Solinski HJ, Mishra SK, Tseng PY, Iwagaki N, Boyle KA, Dickie AC, Kriegbaum MC, Wildner H, Zeilhofer HU, Watanabe M, Riddell JS, Todd AJ, Hoon MA (2018) Circuit dissection of the role of somatostatin in itch and pain. Nat Neurosci 21:707-716.

Kardon AP, Polgár E, Hachisuka J, Snyder LM, Cameron D, Savage S, Cai X, Karnup S, Fan CR, Hemenway GM, Bernard CS, Schwartz ES, Nagase H, Schwarzer C, Watanabe M, Furuta T, Kaneko T, Koerber HR, Todd AJ, Ross SE (2014) Dynorphin acts as a neuromodulator to inhibit itch in the dorsal horn of the spinal cord. Neuron 82:573-586.

Leknes SG, Bantick S, Willis CM, Wilkinson JD, Wise RG, Tracey I (2007) Itch and motivation to scratch: an investigation of the central and peripheral correlates of allergen- and histamine-induced itch in humans. J Neurophysiol 97:415-422.

Liu Q, Tang Z, Surdenikova L, Kim S, Patel KN, Kim A, Ru F, Guan Y, Weng HJ, Geng Y, Undem BJ, Kollarik M, Chen ZF, Anderson DJ, Dong X (2009) Sensory neuron-specific GPCR Mrgprs are itch receptors mediating chloroquine-induced pruritus. Cell 139:1353-1365. 
Liu Y, Abdel Samad O, Zhang L, Duan B, Tong Q, Lopes C, Ji RR, Lowell BB, Ma Q (2010) VGLUT2-dependent glutamate release from nociceptors is required to sense pain and suppress itch. Neuron 68:543-556.

Lo L, Anderson DJ (2011) A Cre-dependent, anterograde transsynaptic viral tracer for mapping output pathways of genetically marked neurons. Neuron 72:938-950.

Ma Q (2010) Labeled lines meet and talk: population coding of somatic sensations. J Clin Invest 120:3773-3778.

Mishra SK, Hoon MA (2013) The cells and circuitry for itch responses in mice. Science 340:968-971.

Mu D, Deng J, Liu KF, Wu ZY, Shi YF, Guo WM, Mao QQ, Liu XJ, Li H, Sun YG (2017) A central neural circuit for itch sensation. Science 357:695699.

Nguyen MQ, Wu Y, Bonilla LS, von Buchholtz LJ, Ryba NJP (2017) Diversity amongst trigeminal neurons revealed by high throughput single cell sequencing. PLoS One 12:e185543.

Papoiu AD, Emerson NM, Patel TS, Kraft RA, Valdes-Rodriguez R, Nattkemper LA, Coghill RC, Yosipovitch G (2014) Voxel-based morphometry and arterial spin labeling fMRI reveal neuropathic and neuroplastic features of brain processing of itch in end-stage renal disease. J Neurophysiol 112:1729-1738

Papoiu AD, Kraft RA, Coghill RC, Yosipovitch G (2015) Butorphanol suppression of histamine itch is mediated by nucleus accumbens and septal nuclei: a pharmacological fMRI study. J Invest Dermatol 135:560-568.

Piggins HD, Antle MC, Rusak B (1995) Neuropeptides phase shift the mammalian circadian pacemaker. J Neurosci 15:5612-5622.

Punnakkal P, von Schoultz C, Haenraets K, Wildner H, Zeilhofer HU (2014) Morphological, biophysical and synaptic properties of glutamatergic neurons of the mouse spinal dorsal horn. J Physiol 592:759-776.
Schmelz M, Schmidt R, Bickel A, Handwerker HO, Torebjörk HE (1997) Specific C-receptors for itch in human skin. J Neurosci 17:8003-8008.

Solorzano C, Villafuerte D, Meda K, Cevikbas F, Bráz J, Sharif-Naeini R, Juarez-Salinas D, Llewellyn-Smith IJ, Guan Z, Basbaum AI (2015) Primary afferent and spinal cord expression of gastrin-releasing peptide: message, protein, and antibody concerns. J Neurosci 35:648-657.

Sun S, Xu Q, Guo C, Guan Y, Liu Q, Dong X (2017) Leaky gate model: intensity-dependent coding of pain and itch in the spinal cord. Neuron 93:840-853.e5.

Sun YG, Chen ZF (2007) A gastrin-releasing peptide receptor mediates the itch sensation in the spinal cord. Nature 448:700-703.

Sun YG, Zhao ZQ, Meng XL, Yin J, Liu XY, Chen ZF (2009) Cellular basis of itch sensation. Science 325:1531-1534.

Usoskin D, Furlan A, Islam S, Abdo H, Lönnerberg P, Lou D, HjerlingLeffler J, Haeggström J, Kharchenko O, Kharchenko PV, Linnarsson S, Ernfors P (2015) Unbiased classification of sensory neuron types by large-scale single-cell RNA sequencing. Nat Neurosci 18:145-153.

Vierow V, Forster C, Vogelgsang R, Dörfler A, Handwerker HO (2015) Cerebral networks linked to itch-related sensations induced by histamine and capsaicin. Acta Derm Venereol 95:645-652.

Wada E, Way J, Lebacq-Verheyden AM, Battey JF (1990) Neuromedin B and gastrin-releasing peptide mRNAs are differentially distributed in the rat nervous system. J Neurosci 10:2917-2930.

Wan L, Jin H, Liu XY, Jeffry J, Barry DM, Shen KF, Peng JH, Liu XT, Jin JH, Sun Y, Kim R, Meng QT, Mo P, Yin J, Tao A, Bardoni R, Chen ZF (2017) Distinct roles of NMB and GRP in itch transmission. Sci Rep 7:15466.

Wang H, Zylka MJ (2009) Mrgprd-expressing polymodal nociceptive neurons innervate most known classes of substantia gelatinosa neurons. J Neurosci 29:13202-13209. 